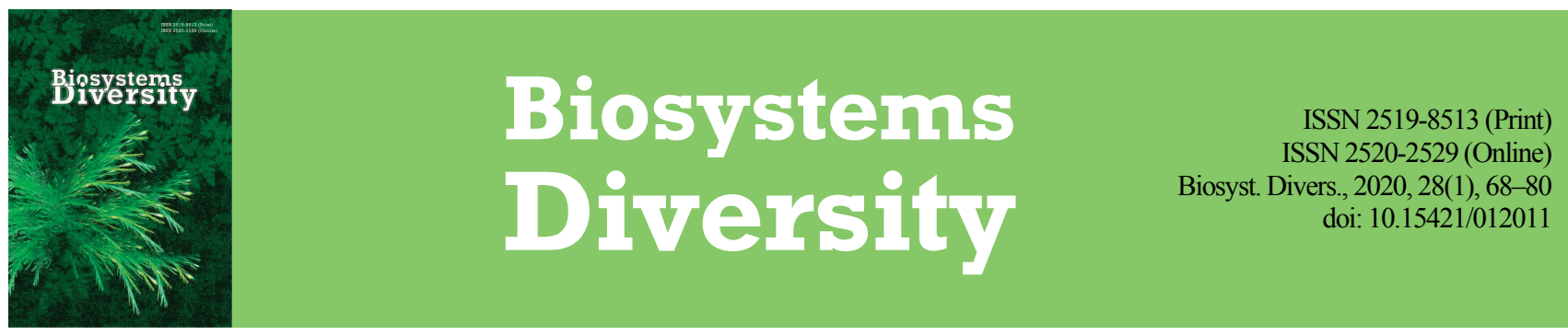

\title{
Syntaxonomy and ecological differentiation of the pioneer vegetation of Ukraine. 1. Classes: Cakiletea maritimae, Ammophiletea, Crithmo-Staticetea, Crypsietea aculeatae, Therosalicornietea
}

\author{
D. V. Dubyna*, T. P. Dziuba*, S. M. Iemelianova*, L. M. Makhynia** \\ *M. G. Kholodny Institute of Botany, National Academy of Sciences of Ukraine, Kyiv, Ukraine \\ **Bogomolets National Medical University, Kyiv, Ukraine
}

Article info

Received 18.01.2020

Received in revised form 10.02 .2020

Accepted 12.02.2020

M. G. Kholodny Institute of Botany, NAS of Ukraine

Tereshchenkivska st., 2

Kyiv, 01004, Ukraine.

Tel.: +38-099-079-01-08

E-mail:ddub@ukr.net

Bogomolets National Medical University,

Pushkinska st., 22,

Kyiv, 01601, Ukraine.

Tel.: +38-063-224-02-18

E-mail:

larisamahin@gmail.com

\begin{abstract}
Dubyna, D. V., Dziuba, T. P., Iemelianova, S. M., \& Makhynia, L. M. (2020). Syntaxonomy and ecological differentiation of the pioneer vegetation of Ukraine. 1. Classes: Cakiletea maritimae, Ammophiletea, Crithmo-Staticetea, Crypsietea aculeatae, Therosalicornietea. Biosystems Diversity, 28(1), 68-80. doi:10.15421/012011

Pioneer plant communities grow on newly-formed ecotopes of coastal and partially continental areas, on patches that have dried after the surface water flooding. They are affected by sea tides, wind-induced effect, salinity, denudation, accumulation and sharp changes in soil humidity. To these factors are added chemical and biological pollution, as well as anthropogenic pressure. Due to these reasons, such plant communities have a specific floristic composition, structure, functional regime and resilience mechanisms, which are different from coenoses of more stable habitats. On the basis of the analysis of more than 600 phytosociological relevés using TWINSPAN modified algorithm the current state of littoral and halophytic classes of pioneer vegetation in Ukraine has been established and their syntaxonomic structure identified. It has been determined that the level of pioneer phytocoenoses diversity in Ukraine is similar to that of Central Europe. The Cakiletea maritimae class is presented by 1 order, 1 alliance, and 4 associations; Ammophiletea - by 1 order, 1 alliance, and 8 associations; Crithmo-Staticetea - by 1 order, 2 alliances, and 3 associations; Crypsietea aculeatae - by 1 order, 3 alliances, and 4 associations, Therosalicornietea - by 1 order, 2 alliances, and 10 associations. Synoptic tables of vegetation classes are given and nine alliances are briefly characterized. Using DCA-ordination analysis of syntaxa in the association-rank level we identified that the main factors of ecological differentiation are soil humidity and aeration as well as the nitrogen content. For the pioneer plant communities, other significant impact factors are the extremality of environmental conditions, their unevenness and non-equilibrium, in particular, the mechanical action of the sea waves, the dynamic processes of denudation and accumulation, salinity, as well as the variability of damping during the year. This research contributes to the identification of the place of the distinguished syntaxa of Ukrainian pioneer vegetation in the European system.
\end{abstract}

Keywords: plant communities; classification; new-formed ecotope; ecological gradients; DCA-ordination; Ukraine.

\section{Introduction}

Pioneer phytocoenoses are the initial producers of organic substances and initiate the development of derivative plant communities; they also have very important biological and ecological functions. Geocomplexes where littoral vegetation is formed are places of origin and conservation of biodiversity. They are also habitats for many plant and animal species, the source of spreading of their genetic material (DNA). Newly-formed ecotopes are perfect migration pathways for alien species. Besides, these territories are the centers of formation of endemic vegetation complexes that are distinguished by genus endemicity, especially in the coastal areas. Considerable variability of environmental factors ensures the exceptional role of pioneer plant communities in the development of adaptation processes. There is a theory that pioneer phytocoenoses will have a faster reaction to global climate changes than other types of vegetation. This difference is due to the predominance of alternative mechanisms of regulation of seasonal cycles in pioneer plants (Sergienko \& Sonina, 2011). Other studies also confirm the hypothesis that the coastal dune vegetation is exposed to local climate influence which has significant effects on both floristic composition and plant community structure. As a result, the coastal phytocoenoses are particularly prone to the effect of possible climate change which may change species composition and distribution as well as disturb the native plant communities' development (Del Vecchio et al., 2018). That is why research on this vegetation type is highly relevant.

The syntaxonomy of pioneer vegetation in Europe has been well developed. In particular, classification schemes and syntaxa characteristics have been determined for the territories of Slovenia (Valachovič, 1995),
France (Bardat et al., 2004), Italy (Biondi et al., 2014), Spain and Portugal (Rivas-Martínez et al., 2001), Bulgaria (Tzonev et al., 2009), Hungary (Borhidi, 2003), Germany (Berg et al., 2004), Romania (Sanda et al., 2008) etc. European high and middle-rank syntaxa are generalized in "Vegetation of Europe..." (Mucina et al., 2016).

In comparison to countries of Western Europe, syntaxonomic structure of the pioneer plant communities in Ukraine has not been fully developed yet. Studies of Cakiletea maritimae, Ammophiletea, Crithmo-Staticetea and Therosalicornietea phytocoenoses has been conducted mainly in the Crimea and the coastal areas of the Black and Azov Sea (Vicherek, 1971; Dubyna et al., 1995, 2003, 2007a; Dubyna \& Neuhäuslová, 2003; Dubyna \& Dziuba, 2005; Vojtyuk, 2005; Tyshchenko, 2006; Korzhenevskiy \& Kvitnitskaya, 2014). The Crypsietea aculeatae class communities, due to their ephemeral character, remain less researched; territorially they are present in the regions of the foreststeppe zone in the basin of Dnieper River (Bairak, 1997; Dziuba et al., 2010). Syntaxomic structure of these classes has been analyzed in some generalizing scientific papers (Tymoshenko, 1999; Korzhenevskiy et al., 2003; Dubyna et al., 2004, 2007b). Brief information about the syntaxa of these classes also has been published in the "Prodrome of the Vegetation of Ukraine" (Dubyna et al., 2019a).

The vegetation of Cakiletea maritimae, Ammophiletea, CrithmoStaticetea, Crypsietea aculeatae and Therosalicornietea classes grows on new-formed ecotopes of coastal and continental areas, on the territories that have dried after surface flooding. They are affected by sea tides, wind-induced effect, salinity, denudation, accumulation and rapid changes in soil humidity. To these factors are added chemical and biological 
pollution, as well as anthropogenic pressure. Due to these reasons, such plant communities have specific floristic composition, structure, functional regime and resilience mechanisms which are different from coenoses of more stable habitats. Today they are in the focus of many ecological problems associated with the large-scale building of hydropower in recent years, afforestation of sandy dunes and melioration of saline soils.

Development of nature conservation in the country and participation of Ukraine in international conventions on the conservation of biodiversity with its involvement in the European process of establishment of a single syntaxomic system in Europe, on the basis of the international scientific cooperation, raise questions about coentotic identification of its vegetation. The paper examines the syntaxonomy of pioneer vegetation of Ukraine and consists of three parts. In the first, we consider the syntaxonomy of the pioneer plant communities of the coastal dunes and halophytic new-formed ecotopes which involves the Cakiletea maritimae, Ammophiletea, Crithmo-Staticetea, Crypsietea aculeatae, Therosalicornietea classes; in the second part we will try to find out the syntaxonomy structure of pioneer vegetation on continental alluvial sands; the third part looks into diversity of annual wetland herbaceous plants on river banks and seasonally flooded territories.

The main aims of this paper are detailing the syntaxonomic structure of pioneer littoral and halophytic vegetation of Ukraine and clarifying its current state. On the base of DCA-ordination analysis - identification of the leading factors of ecological differentiation phytocoenoses. This method is widespread in biology and ecology for quantitative assessment of ecological changes from the geological datasets (CorreaMetrio et al., 2014), studies of vegetation successions (Cao et al., 2019), building of hierarchical syntaxonomic schemes (Çoban \& Willner, 2019; Zhou et al., 2019; Willner et al., 2019), identification of the main ecological factors of plant communities' differentiation (Korolyuk et al., 2018; Lashchinskiy et al., 2019), assessment of the impact of the environment on the floristic richness of a phytocoenosis (Jansons et al., 2016; Yousaf et al., 2016; Slezák et al., 2017) and other tasks. Using this method for studying the differentiation of pioneer vegetation in ecological space by the 12 vectors-ecofactors supplements phytosociological researches and allows us to detect ecosystems' conditions by the indicators of biotic components (Didukh, 2012).

The problem questions about syntaxonomic content of Crypsietea aculeatae and Therosalicornietea classes have been discussed.

\section{Materials and methods}

The studied territory is located in the south part of Ukraine, in particular, on the coastlines of the Black and Azov Sea (for Cakiletea maritimae, Ammophiletea, Crithmo-Staticetea classes), and in the steppe and the southern regions of forest-steppe zone (for Therosalicornietea, Crypsietea aculeatae classes, Fig. 1). Orographically, the major part of the study area is occupied by the Black Sea Lowland. Its surface is mostly flat and has a general slope to the south. The Danube, Dnister and Dnieper rivers form broad arms, and there are lakes-estuaries in their valleys. The Pryazovya territory is a coastal cumulative terrace plain. Orographically it is a lowland with the heights $0.5-40.0 \mathrm{~m}$ above the sea level. Lakes, islands, estuaries, sand-shelly spits and dunes make the relief of this territory very specific.

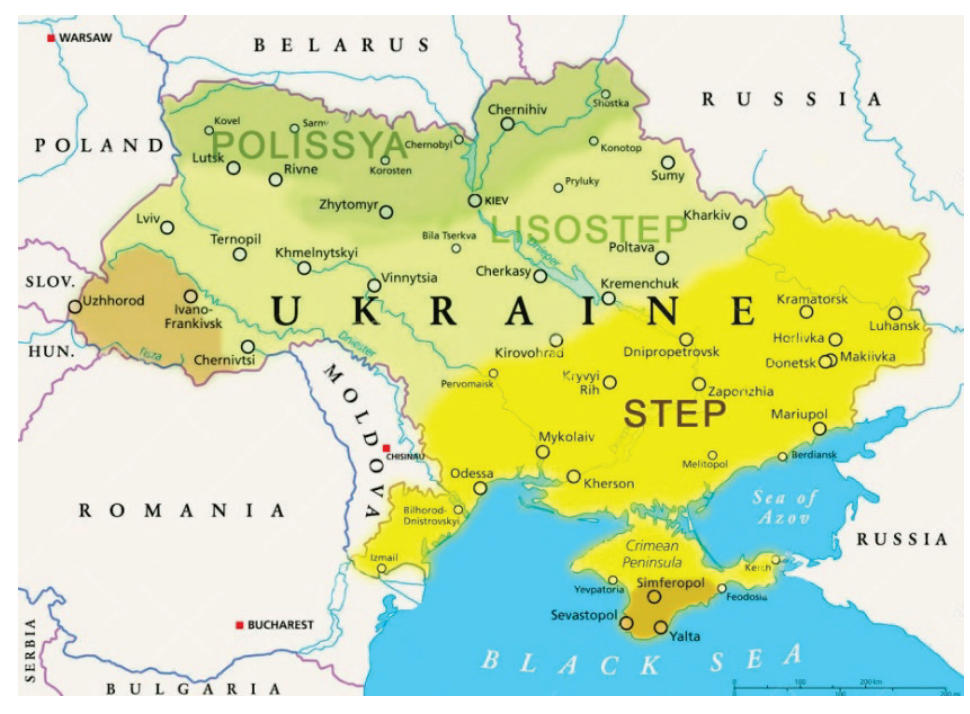

Fig. 1. Location of study area in Ukraine

The location of the Northern Black Sea region and Pryazovya in the south-western part of the Eastern-European plain is marked by a significant amount of solar radiation. The total annual solar radiation fluctuates between $110 \mathrm{kcal} / \mathrm{cm}^{2}$ in the north and $125 \mathrm{kcal} / \mathrm{cm}^{2}$ in the south. The annual radiation balance changes from 50 to $60 \mathrm{kcal} / \mathrm{cm}^{2}$ accordingly. The annual sum of temperatures above $10^{\circ} \mathrm{C}$ is $2,800-3,600{ }^{\circ} \mathrm{C}$. The frostless period lasts for 160-220 days, the period of active vegetation is 160-195 days. The average annual air temperature rises from the northeast to the southwest from $+7.5^{\circ} \mathrm{C}$ to $4.0-11.0^{\circ} \mathrm{C}$; average air temperature in January is from $-7.0^{\circ} \mathrm{C}$ to $-1.0^{\circ} \mathrm{C}$ and average air temperature in July rises from $+21.5^{\circ} \mathrm{C}$ to $+23.0^{\circ} \mathrm{C}$ in the southern direction. The amount of precipitation is from 450 to $350 \mathrm{~mm}$, it is up to $300 \mathrm{~mm}$ in the north part of the study area. Most of the precipitations (60-70\%) fall in the warm period of the year. The characteristic feature of the Northern Black Sea region is high evaporation: $700-800 \mathrm{~mm}$ in the north of the zone and $900-1000 \mathrm{~mm}$ in the south. The humidity index changes from 1.2 to 0.8 , which contributes to the processes of soil salinity (Rudenko, 2007).

Salinity is predominantly sulfate and chloride-sulfate, in the south of the Black Sea Lowland it is chloride. There can be observed the development of secondary soil salinity processes when solonetzes turn back into solonchaks. The reason for this is the epeirogenetic lowering of the coastline, raising the level of saline groundwater on irrigated areas as well as excessive grazing. Typical soils are chestnut, meadow-chestnut, drained, muddy-sand, muddy-shelly as well as solonets and solonchaks.

The northern coastline of the Black and Azov Sea is a moving shelf on the periphery of the continental plate subjected to oscillatory motions of considerable magnitude (Geologicheskaya istoriya, 1980). Moreover, during the last 70 years, a general tendency of annual Ocean level rise by $3.0 \mathrm{~mm}$ is observed, which is associated with the so-called "greenhouse effect" or anthropogenic warming on the planet (Buynevich et al., 2011). During the XX century, the Ocean level rise was $12 \mathrm{~cm}$ at minimum (The State..., 1990), and its rise in the second half of the XXI century is predicted to be more than $10 \mathrm{~cm}$ higher than-contemporary level as a result of on-going global warming.

The original phytosociological relevés, conducted by the authors from 1994 to 2018 according to the Braun-Blanquet approach (Westhoff \& van der Maarel, 1973), have become the main data for syntaxonomy development of the pioneer vegetation of Ukraine, as well as the vegetation plots in the scientific publications mentioned above. The standard size of the plots was mainly 4 x $4 \mathrm{~m}$. In some cases, in particular, on outstretched 
coastlines, the plots were $1 \times 4$ or $2 \times 5 \mathrm{~m}$. We have also followed the requirements of the homogeneous structure of the vegetation cover.

Data arrangement has been made by creating the phytosociological database using TURBOVEG software (Hennekens \& Schaminée, 2001). The phytosociological material interpretation was made in a few steps. In the first stage it was made using Two-Way Indicator Species Analysis (TWINSPAN), in particular, its modified algorithm (Hill, 1979; Tichý, 2002; Roleček et al., 2009). The total dataset of halophytic and littoral pioneer vegetation of Ukraine, that included 6,505 relevés, was processed and divided into smaller groups according to their differences. "Pseudospecies" cut level was $0 \%, 5 \%, 15 \%, 25 \%$. Whittaker's beta diversity index was chosen as a measure of the heterogeneity of the clusters (Whittaker, 1978). After this, every dendrite was analyzed separately and clusters distinguished which by groups of diagnostic species correspond to classes of pioneer vegetation. Within these clusters that included more than 600 relevés, the most homogeneous ones were identified as phytocoenons that have been accorded the rank of alliances. Then after the rejection of some vegetation plots, every cluster was analyzed using PC-ORD software. The Sörensen index was chosen as a measure of the homogeneity of the clusters (Sörensen, 1948), and relevés were grouped by the flexible beta method at 0.25 . The use of PC-ORD algorithm at the final stages of conducting analysis made it possible to identify smaller groups that almost corresponded to the association-rank. The diagnostic species of a syntaxon were determined according to the phi coefficient (Willner et al., 2009), the accepted value is 0.25 . Identification of the received phytocoenons was conducted based on the analysis of their diagnostic species using foreign and Ukrainian publications (Mucina et al., 2016). The method of DCA-ordination (Hill \& Gauch, 1980; ter Braak \& Smilauer, 2015) of R-project (Venables \& Smith, 2008) integrated into a JUICE software package, was used to identify the leading factors of ecological differentiation of plant communities. The ecological parameters were calculated by means of Y. Didukh's phytoindication scales (Didukh, 2011), which allows us to make an ordination analysis by 12 factors: soil humidity $(\mathrm{Hd})$, variability of damping ( $\mathrm{fH}$ ), soil aeration (Ae), nitrogen content in soil (Nt), acidity $(\mathrm{Rc})$, total salt regime $(\mathrm{Sl})$, carbonate content in soil $(\mathrm{Ca})$, thermal climate $(\mathrm{Tm})$, humidity $(\mathrm{Om})$, continental climate $(\mathrm{Kn})$, cryoclimate $(\mathrm{Cr})$, light $(\mathrm{Lc})$. The nomenclature of taxa is given according to "Flora Europea" (Tutin et al., 1964-1980).

\section{Results}

The results of the phytosociological database cluster analysis made it possible to determine the place of the studied pioneer vegetation plant communities among the totality of all phytocoenotic data of halophytic and littoral vegetation of Ukraine (Fig. 2). It has been found out that Ammophiletea plant communities (cluster 13) have the most floristic similarity to the Helichryso-Crucianelletea maritimae phytocoenoses (clusters 14-16), which are often located directly behind them, occupying the hindsurf parts of the seaside shaft and stabilized coastal grey dunes. Also similar to each other are coenoses of the Crithmo-Staticetea (cluster 52) and Cakiletea maritimae (cluster 53) classes, which are located on the sea surf line. The plant communities of Crypsietea aculeatae (cluster 91) and Therosalicornietea (clusters 92, 93) classes also demonstrate a high floristic affinity because they are formed in ecological conditions, similar in several parameters - salt regime, soil humidity, variability of damping, etc. They form a group of dendrites with transitional coenoses to salt meadows of the Festuco-Puccinellietea class (cluster 94). Subsequently, each of the clusters was subjected to the same analysis to obtain phytocoenons of association rank and to identify connections between syntaxa.

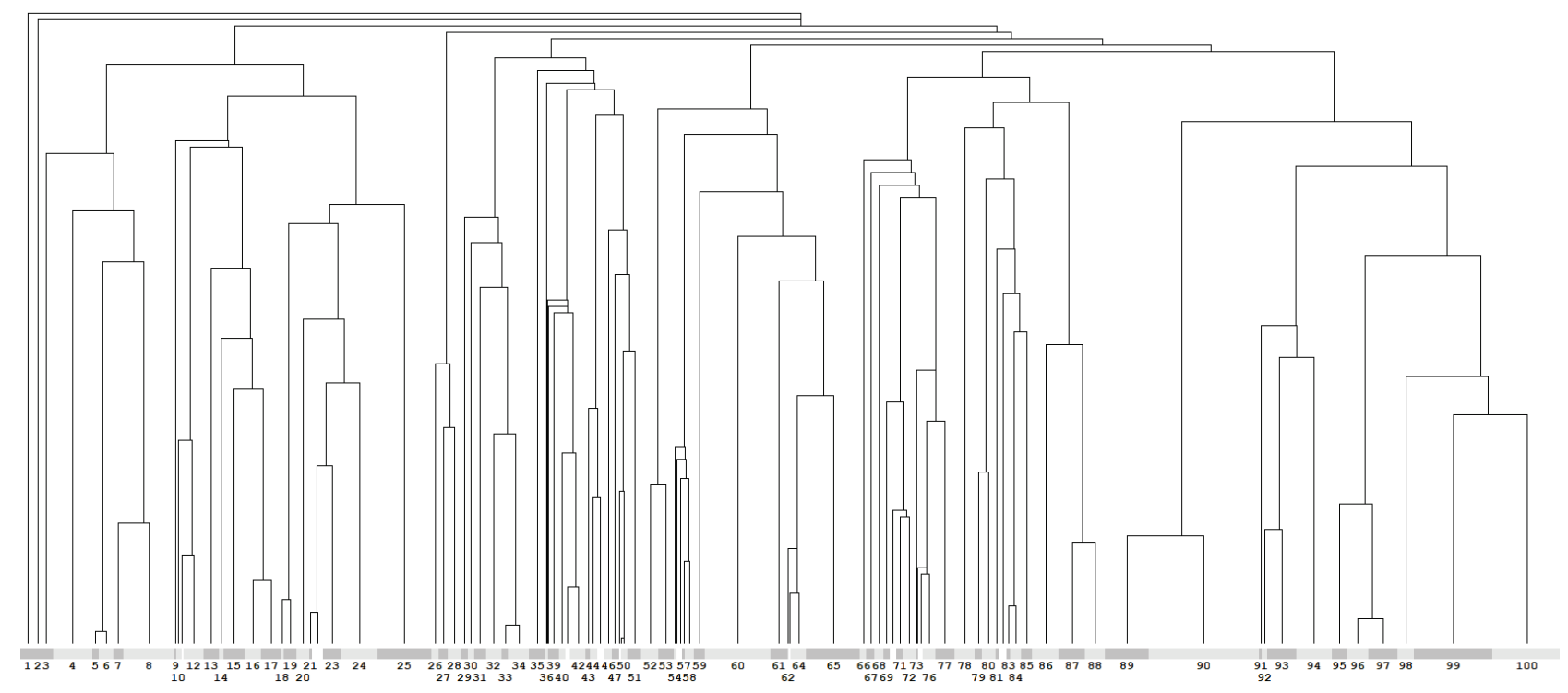

Fig. 2. Hierarchical cluster analysis of relevés (PC-ORD)

Classification Scheme

Cakiletea maritimae Tx. et Preising in Tx. ex Br.-Bl. et Tx. 1952

Thero-Atriplicetalia Pignatti 1953

Cakilion euxinae Géhu et al. 1994

Cakilo euxinae-Euphorbietum peplidis Dubyna, Neuhäuslová et Shelyag-Sosonko 1994

Lactuco tataricae-Cakiletum euxinae Korzhenevsky et Klyukin in Korzhenevsky 2001

Cakilo euxinae-Salsoletum tragi Vicherek 1971

Cakilo euxinae-Salsoletum ruthenicae Vicherek 1971

Ammophiletea Br.-Bl. et Tx. ex Westhoff et al. 1946

Ammophiletalia Br.-Bl. et Tx. ex Westhoff et al. 1946

Elymion gigantei Morariu 1957

Tournefortietum sibiricae Popescu et Sanda 1975

Salsoletum sodae Slavnić 1948

Elymetum gigantei Morariu 1957

Artemisietum arenariae Popescu et Sanda 1975

Asparago levinae-Calamagrostidetum epigei Vicherek 1971 
Centaurea odessanae-Elymetum gigantei Vicherek 1971

Elymo-Astrodaucetum littoralis Korzhenevsky, Volkova et Klyukin in Korzhenevsky 2001

Crambetum maritimae Şerbănescu 1970

Crithmo-Staticetea Br.-Bl. in Br.-Bl. et al. 1952

Crithmo-Staticetalia Molinier 1934

Kochio prostratae-Limonion meyeri Korzhenevsky et Klyukin 1987

Puccinellio distantis-Limonietum meyeri Korzhenevsky et Klyukin 1987

Elytrigio bessarabicae-Lactucion tataricae Korzhenevsky ex Didukh et Mucina in Mucina et al. 2016

Crithmo-Elytrigietum bessarabicae Korzhenevsky et Klyukin in Korzhenevsky 2001

Lactuco tataricae-Elytrigietum bessarabicae Korzhenevsky et Klyukin in Korzhenevsky 2001

Therosalicornietea Tx. in Tx. et Oberd. 1958

Camphorosmo-Salicornietalia Borhidi 1996

Salicornion prostratae Géhu 1992

Salicornietum prostratae Soó 1927

Petrosimonio oppositifoliae-Salicornietum Korzhenevsky et Klyukin in Dubyna et al. 2007

Bassietum hirsutae Şerbănescu 1965

Petrosimonio oppositifoliae-Bassietum sedoidis Shelyag-Sosonko et al. 1989

Ofaisto monandri-Salicornietum Dubyna et Neuhäuslová 2003

Halimionetum pedunculatae Şerbănescu 1965

Suaedion acuminatae Golub et Tchorbadze in Golub 1995 corr. Lysenko et Mucina 2015

Salicornio perennantis-Suaedetum salsae Freitag, Golub et Yuritsyna 2001

Suaedetum salsae Golub et Tchorbadze 1995

Suaedetum confusae Golub et Tchorbadze in Golub 1995

Petrosimonio brachiatae-Artemisietum santonicae Korzhenevsky et Klyukin 1991

Crypsietea aculeatae Vicherek 1973

Crypsietalia aculeatae Vicherek 1973

Cypero-Spergularion salinae Slavnić 1948

Crypsietum aculeatae Wenzl 1934

Heleochloion schoenoidis Br.-Bl. ex Rivas Goday 1956

Heleochloëtum schoenoidis Țopa 1939

Polygono salsuginei-Crypsietum aculeatae Korzhenevsky et Klyukin in Korzhenevsky et al. 1997

Lepidion latifolii Golub et Mirkin in Golub 1995

Cynancho acutae-Lepidietum latifolii Dubyna, Neuhäuslová et Shelyag-Sosonko 1994

The class Cakiletea maritimae combines pioneer plant communities of nitrophilous annual herbs in strandlines of sandy and shingle beaches of the coasts that are formed in the tidal upper boundary of the surf. It includes 4 associations (Fig. 3, Table 1). They are common in the coastal areas of the Black and Azov Sea. The leading factors of territorial differentiation of syntaxa are the intensity of abrasive-accumulative activity of the sea, hydrological conditions, soil type, and its consistency.

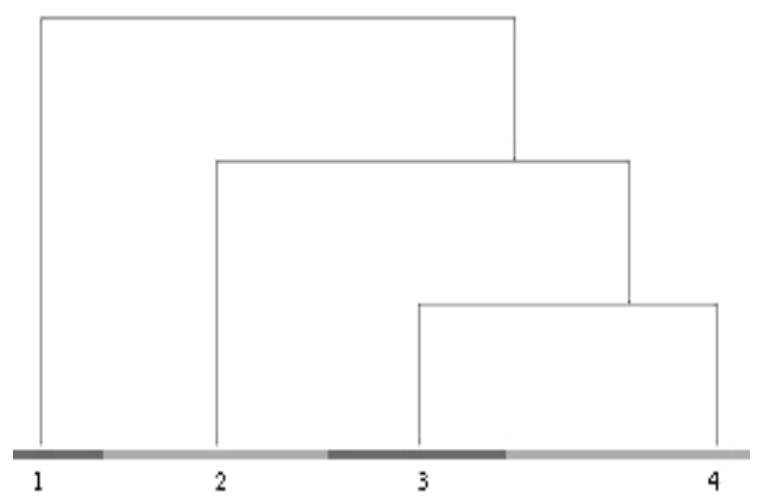

Fig. 3. Hierarchical cluster analysis of Cakiletea maritimae phytosociological relevés: 1 - association Cakilo euxinae-Salsoletum tragi,

2 - association Lactuco tataricae-Cakiletum euxinae, 3 - association Cakilo euxinae-Euphorbietum peplidis, 4 - association Cakilo euxinae-Salsoletum ruthenicae

Alliance Cakilion euxinae includes halo-nitrophilous plant communities of the Black and Azov Sea shores that are formed at the zone of storm washovers. The ordination analyses on the association-rank level made it possible to conclude that the main factors of their differentiation are the variability of damping, soil acidity, nitrogen and carbonate content in the soil, and cryoclimate (Fig. 4). The vectors by these gradients are the closest to the first ordination axis. Additionally, the soil aeration and total salt regime of ecotopes influence the ecological differentiation of coenoses.

Table 1

Synoptic table of the class Cakiletea maritimae

\begin{tabular}{|c|c|c|c|c|}
\hline No. of syntaxa & 1 & 2 & 3 & 4 \\
\hline Number of relevés & 9 & 11 & 10 & 24 \\
\hline 1 & 2 & 3 & 4 & 5 \\
\hline Euphorbia peplis & 58.6 & 16.6 & - & - \\
\hline Astrodaucus littoralis & 39.9 & 14.1 & - & - \\
\hline Leymus racemosus & - & 75.3 & - & - \\
\hline Lactuca tatarica & - & 72.4 & - & - \\
\hline Crambe maritima & 21.7 & 60.2 & - & - \\
\hline Aeluropus littoralis & - & 54.8 & - & - \\
\hline Polygonum aviculare & - & 50.1 & - & 7.4 \\
\hline Atriplex calotheca & - & 47.7 & - & 1.8 \\
\hline Xanthium strumarium s. italicum & - & 44.8 & - & 6.6 \\
\hline Eryngium maritimum & - & 41.9 & - & 9.1 \\
\hline Carex ligerica & - & 37.8 & - & - \\
\hline Convolvulus arvensis & - & 37.8 & - & - \\
\hline Elymus farctus s. bessarabicus & - & 34.9 & - & 13.3 \\
\hline Gypsophila paniculata & - & 26.4 & - & - \\
\hline Silene conica s. subconica & - & 26.4 & - & - \\
\hline Secale sylvestre & - & 26.4 & - & - \\
\hline Clypeola jonthlaspi & - & 26.4 & - & - \\
\hline Elymus repens & - & 26.4 & - & - \\
\hline Hordeum murinum & - & 26.4 & - & - \\
\hline Suaeda altissima & - & 26.4 & - & - \\
\hline Melilotus taurica & - & 26.4 & - & - \\
\hline Melilotus alba & - & 26.4 & - & - \\
\hline Puccinellia distans & - & 26.4 & - & - \\
\hline Aster tripolium & - & 26.4 & - & - \\
\hline Salsola kali s. tragus & - & - & 86.6 & - \\
\hline Atriplex prostrata & - & - & 79.8 & - \\
\hline Cynodon dactylon & - & - & - & 44.7 \\
\hline Cynanchum acutum & - & - & - & 36.1 \\
\hline Glaucium flavum & - & - & - & 31.1 \\
\hline Argusia sibirica & - & - & - & 31.1 \\
\hline Limonium gmelinii & - & - & - & 31.1 \\
\hline Verbascum pinnatifidum & - & - & - & 25.3 \\
\hline Aegilops cylindrica & - & - & - & 25.3 \\
\hline Aegilops neglecta & - & - & - & 25.3 \\
\hline
\end{tabular}




\begin{tabular}{|c|c|c|c|c|}
\hline 1 & 2 & 3 & 4 & 5 \\
\hline Bromus tectorum & - & - & - & 25.3 \\
\hline Salsola kali & - & 42.2 & - & 63.2 \\
\hline Leymus racemosus s. sabulosus & - & - & 24.7 & 23.4 \\
\hline Gypsophila perfoliata & - & - & - & 17.8 \\
\hline Asparagus litoralis & - & - & - & 17.8 \\
\hline Rapistrum rugosum & - & - & - & 17.8 \\
\hline Thlaspi perfoliatum & - & - & - & 17.8 \\
\hline Eryngium campestre & - & - & - & 17.8 \\
\hline Bassia sedoides & - & - & - & 17.8 \\
\hline Centaurea arenaria s. odessana & - & - & - & 17.8 \\
\hline Atriplex tatarica & - & - & - & 17.8 \\
\hline Silene densiflora & - & - & - & 17.8 \\
\hline Aster linosyris & - & - & - & 17.8 \\
\hline Cichorium intybus & - & - & - & 17.8 \\
\hline Cerastium fontanum s. vulgare & - & - & - & 17.8 \\
\hline Elaeagnus angustifolia & - & - & - & 17.8 \\
\hline Elymus nodosus & - & - & - & 17.8 \\
\hline Medicago rigidula & - & - & - & 17.8 \\
\hline Artemisia tschernieviana & - & - & 11.0 & 17.2 \\
\hline Cakile maritima s. euxina & - & 9.8 & 9.8 & 9.8 \\
\hline Ephedra distachya & - & 18.6 & - & 2.7 \\
\hline Centaurea apiculata s. adpressa & - & 18.6 & - & 2.7 \\
\hline Salicornia prostrata & - & 18.6 & - & 2.7 \\
\hline Erysimum repandum & - & - & 20.2 & 2.0 \\
\hline Polygonum maritimum & - & 19.6 & 24.7 & - \\
\hline
\end{tabular}

Notes: numbers mark syntaxa: 1 - Cakilo euxinae-Euphorbietum peplidis; 2 - Lactuco tataricae-Cakiletum euxinae; 3 - Cakilo euxinae-Salsoletum tragi; 4-Cakilo euxinae-Salsoletum ruthenicae.

To Ammophiletea class belong the plant communities of coastal mobile sand dunes of the Black and Azov Sea shores. It represented by 8 associations (Fig. 5, Table 2). The phytocoenoses of the class are extended to the most dynamic relief forms that are called avandunes and are always on the transformation stage. They affected by sand blowing from already formed landforms as well as its accumulation from lower located beach areas. Alliance Elymion gigantei includes plant communities of the seaside shaft and coastal white dunes. Their regional specifics become apparent at the associations and subassociations-rank levels and are connected with the intensity of abrasive-accumulative activity of the sea, eolian processes as well as its place in landscape.

The results of ordination of syntaxa within the class have demonstrated that the main factor that identifies its ecological differentiation is the variability of damping, the vector by which it almost aligns with the first ordination axis. The belonging this gradient to the leading ones is determined by coastal location of the plant communities in the zone of regular wind-driven pressure of sea waves (Fig. 6).

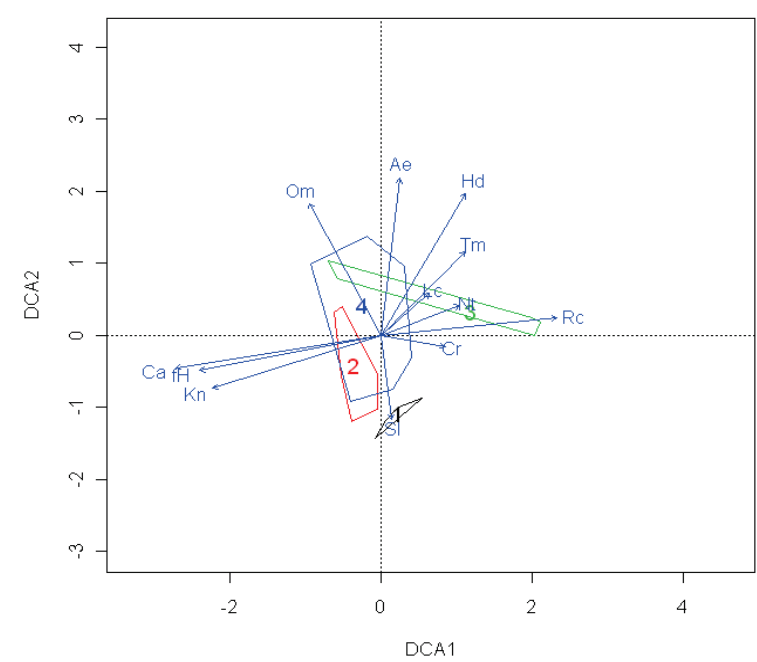

Fig. 4. Results of ordination analysis of the Cakiletea maritimae associations: the numbers mark syntaxa: 1 -Cakilo euxinae-Euphorbietum peplidis; 2 - Lactuco tataricae-Cakiletum euxinae; 3 - Cakilo euxinaeSalsoletum tragi; 4-Cakilo euxinae-Salsoletum ruthenicae; here and after the Y. Didukh's scale (2011) have been used: Hd - soil humidity; $\mathrm{Nt}$ - nitrogen content in soil; $\mathrm{Ca}$ - carbonate content in soil; $\mathrm{Rc}-$ soil acidity; $\mathrm{Sl}$ - total salt regime; $\mathrm{fH}$ - variability of damping; $\mathrm{Ae}$ - soil aeration; Lc - light; $\mathrm{Kn}$ - continental climate; Om - humidity; Tm thermal climate; $\mathrm{Cr}$ - cryoclimate; DCA1, DCA2 - ordination axis

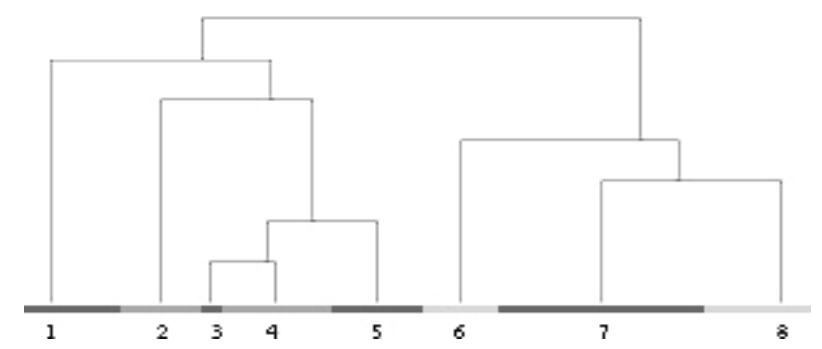

Fig. 5. Hierarchical cluster analysis of Ammophiletea phytosociological relevés: 1 -association Tournefortietum sibiricae, 2-Salsoletum sodae, 3 - association Artemisietum arenariae, 4-association Elymetum gigantei, 5 -association Crambetum maritimae, 6 -association Asparago levinae-Calamagrostietum epigei, 7 - association Centaurea odessanaeElymetum gigantei, 8 - association Elymo-Astrodancetum littoralis

Table 2

Synoptic table of the class Ammophiletea

\begin{tabular}{|c|c|c|c|c|c|c|c|c|}
\hline No. of syntaxa & 1 & 2 & 3 & 4 & 5 & 6 & 7 & 8 \\
\hline Number of relevés & 25 & 10 & 20 & 6 & 10 & 13 & 17 & 14 \\
\hline 1 & 2 & 3 & 4 & 5 & 6 & 7 & 8 & 9 \\
\hline Elymus farctus & 88.2 & - & - & - & - & - & - & - \\
\hline Elymus repens s. elongatiformis & 42.4 & - & - & - & - & - & - & - \\
\hline Euphorbia seguierana & 37.8 & - & - & - & - & - & - & - \\
\hline Helichrysum arenarium & 37.8 & - & - & - & - & - & - & - \\
\hline Festuca beckeri & 36.7 & - & 4.1 & - & - & - & - & - \\
\hline Asparagus maritimus & - & 94.2 & - & - & - & - & - & - \\
\hline Calamagrostis epigejos & - & 87.8 & - & - & 4.6 & - & - & - \\
\hline Medicago sativa s. falcata & 19.0 & 63.1 & - & - & - & - & - & - \\
\hline Syrenia montana & - & 36.3 & - & - & - & - & - & 23.1 \\
\hline Galium tenderiense & 17.4 & 31.5 & - & - & - & - & 5.7 & - \\
\hline Polygonum maritimum & - & - & 60.7 & - & - & - & - & - \\
\hline Polygonum aviculare & - & - & 60.7 & - & - & - & - & - \\
\hline Salsola kali & - & - & 43.1 & - & 15.8 & - & - & - \\
\hline Cerastium pumilum & - & - & 36.6 & - & - & - & - & - \\
\hline Plantago arenaria & - & - & 35.8 & - & - & - & 5.7 & - \\
\hline Xanthium strumarium s. italicum & - & - & 34.7 & - & - & - & - & 7.8 \\
\hline Holosteum umbellatum & - & - & 29.8 & - & - & - & - & - \\
\hline Erophila verna & - & - & 29.8 & - & - & - & - & - \\
\hline Carduus uncinatus & - & - & 29.8 & - & - & - & - & - \\
\hline Alyssum turkestanicum & - & - & 29.8 & - & - & - & - & - \\
\hline Suaeda prostrata & - & - & - & 90.2 & - & - & - & - \\
\hline
\end{tabular}




\begin{tabular}{|c|c|c|c|c|c|c|c|c|}
\hline 1 & 2 & 3 & 4 & 5 & 6 & 7 & 8 & 9 \\
\hline Puccinellia distans & - & - & - & 90.2 & - & - & - & - \\
\hline Argusia sibirica & - & - & - & 85.3 & - & - & - & - \\
\hline Salicornia prostrata & - & - & - & 79.8 & - & - & - & - \\
\hline Polygonum arenarium s. arenarium & - & - & - & 63.2 & - & 12.7 & - & - \\
\hline Bromus squarrosus & - & - & - & 59.9 & - & - & 5.7 & - \\
\hline Melilotus alba & - & 13.1 & - & 53.1 & - & - & - & 18.8 \\
\hline Bassia hirsuta & - & - & - & 30.5 & - & - & - & 9.3 \\
\hline Artemisia tschernieviana & 3.3 & - & - & - & 84.4 & - & - & - \\
\hline Elymus nodosus & - & - & - & - & 60.7 & - & - & - \\
\hline Amorpha fruticosa & - & - & - & - & 42.4 & - & - & - \\
\hline Petasites spurius & - & - & - & - & 42.4 & - & - & - \\
\hline Salix viminalis & - & - & - & - & 42.4 & - & - & - \\
\hline Elymus farctus s. bessarabicus & - & - & 12.4 & - & 32.3 & - & - & - \\
\hline Picris hieracioides s. hieracioides & - & - & 1.7 & - & 30.4 & 6.9 & - & - \\
\hline Elaeagnus angustifolia & - & - & - & - & 29.8 & - & - & - \\
\hline Hippophae rhamnoides & - & - & - & - & 29.8 & - & - & - \\
\hline Asparagus brachyphyllus & - & - & - & - & 29.8 & - & - & - \\
\hline Artemisia santonicum & - & - & - & - & - & 53.3 & 13.5 & - \\
\hline Limonium latifolium & - & - & - & - & - & 52.9 & - & - \\
\hline Elymus elongatus & - & - & - & - & - & 34.7 & 13.7 & - \\
\hline Plantago maritima & - & - & - & - & - & 26.1 & - & - \\
\hline Cakile maritima & - & - & - & - & - & 26.1 & - & - \\
\hline Euphorbia paralias & - & - & - & - & - & 26.1 & - & - \\
\hline Seseli campestre & - & - & - & - & - & - & 51.7 & - \\
\hline Elymus uralensis s. viridiglumis & - & - & - & - & - & - & 32.3 & - \\
\hline Petasites albus & - & - & - & - & - & - & 32.3 & - \\
\hline Erysimum repandum & - & - & - & - & - & - & 32.3 & - \\
\hline Artemisia campestris s. campestris & - & - & - & - & - & - & - & 62.9 \\
\hline Linaria genistifolia & - & 18.9 & - & - & - & - & - & 57.2 \\
\hline Secale sylvestre & - & - & - & - & 1.7 & - & 4.1 & 45.8 \\
\hline Suaeda confusa & - & - & - & - & - & - & - & 43.9 \\
\hline Chondrilla juncea & - & - & - & - & - & - & - & 43.9 \\
\hline Crambe maritima & 18.6 & 17.1 & 24.6 & 1.9 & - & - & - & 39.8 \\
\hline Xanthium strumarium & - & - & - & - & - & 24.6 & 1.3 & 39.4 \\
\hline Lepidium latifolium & - & - & - & - & - & - & 5.1 & 37.5 \\
\hline Convolvulus arvensis & - & - & - & - & - & - & - & 35.7 \\
\hline Chenopodium album & - & - & - & - & - & - & - & 35.7 \\
\hline Bromus tectorum & - & - & - & - & - & - & 8.1 & 28.4 \\
\hline Rumex confertus & - & - & - & - & - & - & - & 25.1 \\
\hline Agropyron cristatum s. pectinatum & - & - & - & - & - & - & - & 25.1 \\
\hline Atriplex tatarica & - & - & - & - & - & - & - & 25.1 \\
\hline Glaucium flavum & - & - & - & - & - & - & - & 25.1 \\
\hline Amaranthus blitoides & - & - & - & - & - & - & - & 25.1 \\
\hline Althaea cannabina & - & - & - & - & - & - & - & 25.1 \\
\hline Scorzonera parviflora & - & - & - & - & - & - & - & 25.1 \\
\hline Elymus repens s. pseudocaesius & - & - & - & - & - & - & - & 25.1 \\
\hline Gypsophila fastigiata & - & - & - & - & - & - & - & 25.1 \\
\hline Matricaria perforata & - & - & - & - & - & - & - & 25.1 \\
\hline Marrubium peregrinum & - & - & - & - & - & - & - & 25.1 \\
\hline Melilotus officinalis & - & - & - & - & - & - & - & 25.1 \\
\hline Centaurea arenaria s. odessana & 49.9 & - & - & - & 18.1 & - & - & 42.0 \\
\hline Seseli arenarium & 41.8 & 28.4 & - & - & - & - & - & - \\
\hline Consolida paniculata & 34.3 & 31.5 & - & - & - & - & - & - \\
\hline Cynanchum acutum & 31.5 & 57.9 & - & - & - & - & 23.9 & - \\
\hline Lactuca tatarica & 27.9 & 37.0 & 10.5 & - & - & - & - & - \\
\hline Astrodaucus littoralis & - & - & 51.5 & - & - & - & - & 38.8 \\
\hline Cakile maritima s. euxina & - & - & 38.2 & 54.3 & - & - & - & 2.5 \\
\hline Euphorbia peplis & - & - & 26.8 & - & - & 35.3 & - & - \\
\hline Salsola soda & - & - & - & 37.4 & - & 66.7 & - & - \\
\hline Gypsophila perfoliata & - & - & - & 28.0 & 28.0 & - & - & 7.8 \\
\hline Leymus racemosus & 47.9 & 47.9 & 47.9 & - & - & - & - & - \\
\hline Leymus racemosus s. sabulosus & - & - & - & 8.5 & 33.9 & 16.3 & 33.9 & 33.9 \\
\hline Galium humifusum & - & - & 22.8 & - & - & - & - & 21.4 \\
\hline Seseli tortuosum & - & - & 10.8 & - & - & - & - & 17.4 \\
\hline Linum austriacum & - & - & 10.8 & - & - & - & - & 17.4 \\
\hline Verbascum pinnatifidum & - & - & 10.8 & - & - & - & - & 17.4 \\
\hline Falcaria vulgaris & - & - & 10.8 & - & - & - & - & 17.4 \\
\hline Alyssum hirsutum & - & - & 20.5 & - & - & - & - & 13.1 \\
\hline Cichorium intybus & - & - & 20.5 & - & - & - & - & 13.1 \\
\hline Syrenia cana & - & - & - & - & 20.5 & - & - & 13.1 \\
\hline Astragalus onobrychis & - & - & 20.5 & - & - & - & - & 13.1 \\
\hline Eryngium campestre & - & - & 5.1 & - & 16.7 & - & - & 10.1 \\
\hline Salsola kali s. tragus & - & - & - & 11.6 & - & 20.4 & 4.9 & 8.3 \\
\hline Carex ligerica & 22.0 & - & 1.4 & - & - & - & 3.1 & 5.4 \\
\hline Conyza canadensis & - & - & - & 22.4 & - & - & 13.4 & 4.9 \\
\hline Eryngium maritimum & 19.6 & - & 10.3 & 23.0 & - & - & - & - \\
\hline Orobanche coerulescens & 18.8 & - & - & - & - & - & - & - \\
\hline
\end{tabular}




\begin{tabular}{|c|c|c|c|c|c|c|c|c|}
\hline 1 & 2 & 3 & 4 & 5 & 6 & 7 & 8 & 9 \\
\hline Thymus dimorphus & - & - & 21.0 & - & - & - & - & - \\
\hline Phragmites australis & - & - & 21.0 & - & - & - & - & - \\
\hline Lepidium perfoliatum & - & - & 21.0 & - & - & - & - & - \\
\hline Asperula tenella & - & - & 21.0 & - & - & - & - & - \\
\hline Coronilla varia & - & - & 21.0 & - & - & - & - & - \\
\hline Medicago lupulina & - & - & 21.0 & - & - & - & - & - \\
\hline Papaver dubium & - & - & 21.0 & - & - & - & - & - \\
\hline Senecio vernalis & - & - & 21.0 & - & - & - & - & - \\
\hline Holosteum umbellatum s. glutinosum & - & - & 21.0 & - & - & - & - & - \\
\hline Arenaria serpyllifolia & - & - & 21.0 & - & - & - & - & - \\
\hline Teucrium polium & - & - & 21.0 & - & - & - & - & - \\
\hline Centaurea arenaria s. majorovii & - & - & 21.0 & - & - & - & - & - \\
\hline Aeluropus littoralis & - & - & 21.0 & - & - & - & - & - \\
\hline Plantago lanceolata & - & - & 21.0 & - & - & - & - & - \\
\hline Erysimum diffusum & - & - & 21.0 & - & - & - & - & - \\
\hline Myagrum perfoliatum & - & - & 21.0 & - & - & - & - & - \\
\hline Sisymbrium polymorphum & - & - & - & - & - & - & 22.8 & - \\
\hline Tragopogon brevirostris s. brevirostris & - & - & - & - & - & - & 22.8 & - \\
\hline Elymus repens & - & - & - & - & - & - & 22.8 & - \\
\hline Linum perenne & - & - & - & - & - & - & 22.8 & - \\
\hline Artemisia salsoloides & - & - & - & - & - & - & 22.8 & - \\
\hline Inula britannica & - & - & - & - & - & - & 22.8 & - \\
\hline Cynodon dactylon & - & - & - & - & - & - & 22.8 & - \\
\hline Polygonum species & - & - & - & - & - & - & 22.8 & - \\
\hline Ephedra distachya & 18.1 & - & - & - & - & - & 12.0 & - \\
\hline Picris hieracioides & - & - & - & - & 21.7 & - & 10.6 & - \\
\hline Centaurea apiculata s. adpressa & - & - & - & - & 21.7 & - & 10.6 & - \\
\hline
\end{tabular}

Notes: the numbers marked syntaxa: 1 - Centaureo odessanae-Elymetum gigantei; 2 -Asparago levinae-Calamagrostidetum epigei; 3 - Elymo-Astrodaucetum littoralis; 4-Tournefortietum sibiricae; 5-Artemisietum arenariae; 6-Salsoletum sodae; 7 -Elymetum gigantei; 8-Crambetum maritimae.

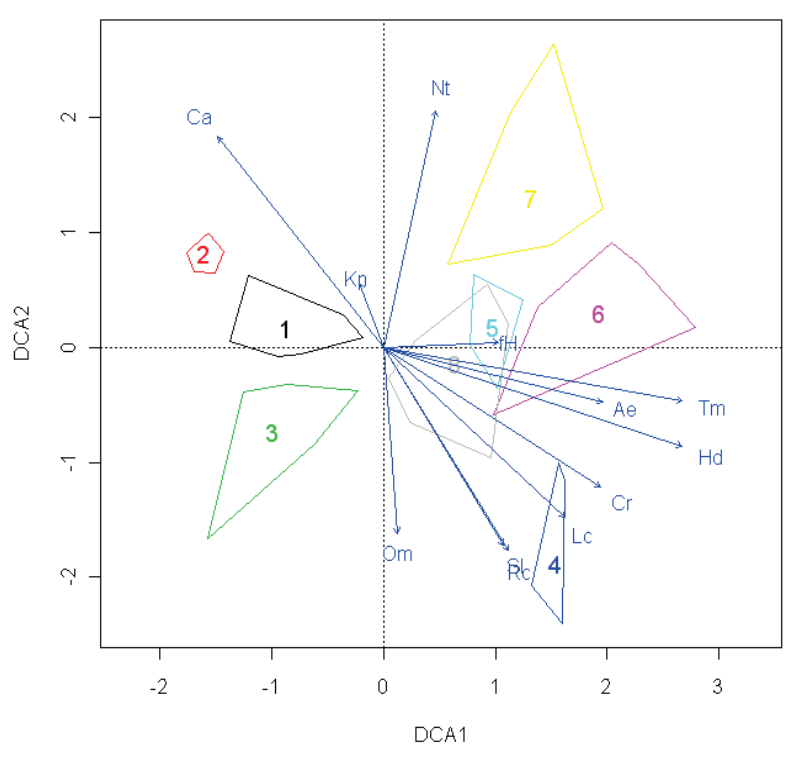

Fig. 6. Results of ordination analysis of the Ammophiletea associations: the numbers marked syntaxa: 1 -Centaureo odessanae-Elymetum gigantei; 2 - Asparago levinae-Calamagrostidetum epigei;

3 -Elymo-Astrodancetum littoralis; 4 - Tournefortietum sibiricae;

5-Artemisietum arenariae; 6-Salsoletum sodae; 7-Elymetum gigantei; 8 -Crambetum maritimae

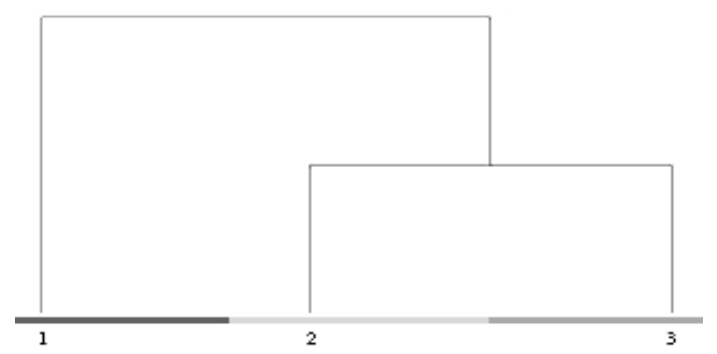

Fig. 7. Hierarchical cluster analysis of Crithmo-Staticetea phytosociological relevés: 1 -association Puccinellio distantis-Limonietum meyeri, 2 - association Crithmo-Elytrigietum bessarabicae, 3 - association Lactuco tataricae-Elytrigietum bessarabicae
The class Crithmo-Staticetea includes rocky and pebble beaches plant communities of the Black Sea abrasive shores that are formed by the influence of saline splatters. It contains 3 associations (Fig. 7, Table 3). The main factors of its territorial differentiation are relief of territory, soil humidity, total salt regime and structure of the substrate. Alliance Kochio prostratae-Limonion meyeri includes phytocoenoses of active and non-active cliffs that consist of limestones on the Kerch Peninsula of Crimea. Alliance Elytrigio bessarabicae-Lactucion tataricae this is plant communities of cliffs, rocky and pebble beaches that are composed of dense carbonates and are widespread on the Eastern coast of the Crimea.

Table 3

Synoptic table of the class Crithmo-Staticetea

\begin{tabular}{|c|c|c|c|}
\hline No. of syntaxa & 1 & 2 & 3 \\
\hline Number of relevés & 10 & 33 & 9 \\
\hline 1 & 2 & 3 & 4 \\
\hline Bassia prostrata & 95.6 & - & - \\
\hline Holosteum umbellatum & 85.3 & - & - \\
\hline Puccinellia distans & 85.3 & - & - \\
\hline Goniolimon tataricum & 78.0 & - & - \\
\hline Halimione verrucifera & 70.7 & - & - \\
\hline Puccinellia festuciformis s. convoluta & 63.2 & - & - \\
\hline Medicago sativa s. falcata & 63.2 & - & - \\
\hline Allium flavum s. tauricum & 55.5 & - & - \\
\hline Poa bulbosa & 55.5 & - & - \\
\hline Bromus hordeaceus s. hordeaceus & 55.5 & - & - \\
\hline Ephedra distachya & 47.1 & - & - \\
\hline Limonium latifolium & 47.1 & - & - \\
\hline Bromus tectorum & 42.9 & - & - \\
\hline Plantago lanceolata & 37.8 & - & - \\
\hline Aster linosyris & 37.8 & - & - \\
\hline Limonium meyeri & 35.0 & - & - \\
\hline Cynanchum acutum & 29.6 & - & 23.2 \\
\hline Asparagus brachyphyllus & 26.3 & - & - \\
\hline Taraxacum erythrospermum & 26.3 & - & - \\
\hline Crithmum maritimum & - & 71.2 & - \\
\hline Seseli dichotomum & - & 50.0 & - \\
\hline Argusia sibirica & - & 50.0 & - \\
\hline Gypsophila muralis & - & 47.4 & - \\
\hline Senecio bicolor & - & 44.7 & - \\
\hline Euphorbia paralias & - & 41.9 & - \\
\hline Seseli gummiferum & - & 39.0 & - \\
\hline Capparis spinosa & - & 39.0 & - \\
\hline Dactylis glomerata s. hispanica & - & 35.9 & - \\
\hline
\end{tabular}




\begin{tabular}{|c|c|c|c|}
\hline 1 & 2 & 3 & 4 \\
\hline Artemisia santonicum & - & 31.8 & - \\
\hline Trachomitum sarmatiense & - & 29.0 & - \\
\hline Parietaria lusitanica s. serbica & - & 29.0 & - \\
\hline Bassia laniflora & - & 25.0 & - \\
\hline Equisetum ramosissimum & - & 25.0 & - \\
\hline Dorycnium pentaphyllum s. herbaceum & - & 25.0 & - \\
\hline Galium humifusum & - & - & 53.9 \\
\hline Atriplex calotheca & - & 9.2 & 51.1 \\
\hline Cakile maritima s. euxina & - & - & 50.0 \\
\hline Aeluropus littoralis & - & - & 50.0 \\
\hline Phragmites australis & - & - & 44.2 \\
\hline Astrodaucus littoralis & - & 14.7 & 41.1 \\
\hline Galium spurium & - & - & 40.0 \\
\hline Alyssum hirsutum & - & - & 40.0 \\
\hline Crambe maritima & - & 8.8 & 38.2 \\
\hline Crambe aspera & - & - & 35.2 \\
\hline Asparagus litoralis & - & - & 35.2 \\
\hline Marrubium peregrinum & - & - & 35.2 \\
\hline Cichorium intybus & - & 8.8 & 31.9 \\
\hline Melica ciliata s. monticola & - & - & 31.0 \\
\hline Euphorbia peplis & - & - & 31.0 \\
\hline Arthonia calcicola & - & - & 27.7 \\
\hline Gypsophila paniculata & - & - & 27.7 \\
\hline Hordeum murinum & - & - & 27.7 \\
\hline Elymus repens & - & - & 27.7 \\
\hline Xanthium strumarium & - & - & 27.7 \\
\hline Convolvulus arvensis & - & - & 27.7 \\
\hline Cardaria draba & - & - & 27.3 \\
\hline Lactuca tatarica & - & 27.7 & 58.2 \\
\hline Aster tripolium & - & 1.0 & 16.4 \\
\hline Glaucium flavum & - & 13.5 & 15.6 \\
\hline Elymus farctus s. bessarabicus & 7.1 & - & 7.1 \\
\hline Melilotus alba & - & - & 3.7 \\
\hline Centaurea solstitialis & - & 14.3 & - \\
\hline Artemisia austriaca & - & 14.3 & - \\
\hline Astragalus glaucus & - & 14.3 & - \\
\hline Sonchus asper & - & 20.3 & - \\
\hline Convolvulus lineatus & - & 20.3 & - \\
\hline Centaurea arenaria s. odessana & - & 20.3 & - \\
\hline Lappula squarrosa s. squarrosa & - & 20.3 & - \\
\hline Cirsium arvense & - & 20.3 & - \\
\hline Lactuca saligna & - & 20.3 & - \\
\hline Eryngium maritimum & - & 20.3 & - \\
\hline
\end{tabular}

Note: the numbers marked syntaxa: 1 -Puccinellio distantis-Limonietum meyeri; 2 - Crithmo-Elytrigietum bessarabicae; 3 - Lactuco tataricae-Elytrigietum bessarabicae.

According to the results of the ordination of Crithmo-Staticetea plant communities (Fig. 8) it has been found that their differentiation in the hyperspace of abiotic factors occurs along the gradient of ecotope light regime as well as climate aridity-humidity and soil humidity.

The class Therosalicornietea combines pioneer plant communities of annual halophyte succulents on wet, periodically flooded ecotopes with solonetzes and solonchaks. They are common in the steppe and in the south of the forest-steppe zone. They are more frequent in the territories where the sea has a direct influence, local depressions (pods), on flat areas near permanent and temporary water reservoirs, estuary shores (coast of the Black and Azov Sea, Prysyvashya). In the continental regions, these phytocoenoses grow on accumulative types of landforms lowlands, river terraces and littoral zone of lakes (left bank river terraces of the Middle Dnieper). Also, Therosalicornietea plant communities are found on saline soils in the places of salt extraction in the Pre-Carpathian region. The class includes 10 associations (Fig. 9, Table 4). Coastal phytocoenoses (especially in Prysyvashya) are characterized by the predominance of hyper-halophytic species within their coenofloras, continental ones (on the left bank of the Dnieper River) - by the prevalence of salt meadow species. Ofaisto monandri-Salicornietum and Petrosimonio oppositifoliae-Salicornietum are endemic associations confined by the territory of Prysyvashya. Estuaries of large rivers (Dnieper, Dnister, Danube) define the territorial specificity because the water regime does not promote the spread of Therosalicornietea plant communities on those territories, as well as coastal spits of the Azov Sea because of their geomorphological peculiarities and more continental climate in comparison to the Black Sea Lowland. The Crimean Peninsula has phytocoenotic uniqueness where such associations as Lepidietum crassifoliae and Petrosimonio brachiatae-Artemisietum santonicae are described. The leading factors of territorial differentiation of syntaxa are relief of ecotopes, periods of offshore flooding, total salt regime, as well as their structure. The alliance Salicornion prostratae includes pioneer vegetation of the lowland territories on the seashores with muddy soils, on coastal zones of estuaries and on the salinized areas in the floodplains of rivers. Thero-Suaedion alliance represents terrophytic succulent vegetation of salinized wet territories of the seashores with the predominance of species of the genus Suaeda.

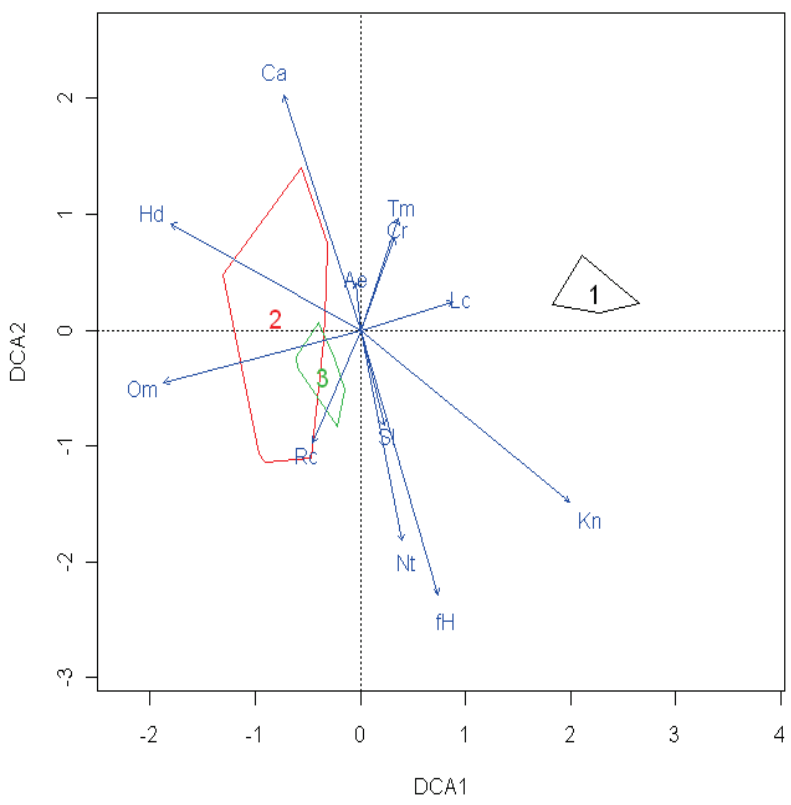

Fig. 8. Results of ordination analysis of the Crithmo-Staticetea associations: the numbers marked syntaxa: 1 -Puccinellio distantis-Limonietum meyeri; 2 - Crithmo-Elytrigietum bessarabicae 3 -Lactuco tataricae-Elytrigietum bessarabicae

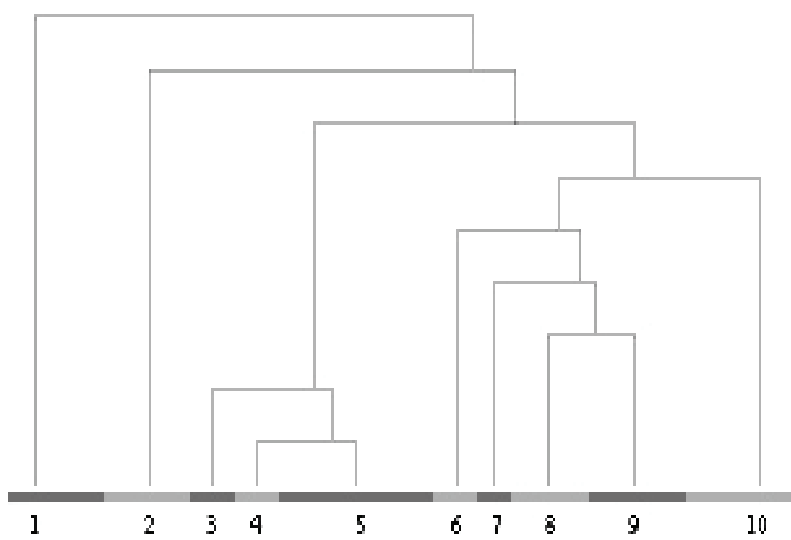

Fig. 9. Hierarchical cluster analysis of Therosalicornietea phytosociological relevés: 1 - association Petrosimonio brachiataeArtemisietum santonicae, 2 - association Petrosimonio oppositifoliaeBassietumsedoidis, 3 - association Suaedetum salsae, 4 - association Salicornio perennantis-Suaedetum salsae, 5 - association Ofaisto monandri-Salicornietum, 6-association Bassietum hirsutae,

7 - association Suaedetum confusae, 8 - association Salicornietum prostratae, 9-association Petrosimonio oppositifoliae-Salicornietum, 10 - association Halimionetum pedunculatae

DCA-ordination of Therosalicornietea class vegetation has demonstrated that their ecological distribution is mainly determined by continental climate (Fig. 10). The additional factors of ecological differentiation are the total salt regime, acidity as well as cryoclimate. 
Table 4

Synoptic table of the class Therosalicornietea

\begin{tabular}{|c|c|c|c|c|c|c|c|c|c|c|}
\hline No.of syntaxa & 1 & 2 & 3 & 4 & 5 & 6 & 7 & 8 & 9 & 10 \\
\hline Number of relevés & 150 & 6 & 11 & 16 & 37 & 11 & 54 & 6 & 14 & 18 \\
\hline 1 & 2 & 3 & 4 & 5 & 6 & 7 & 8 & 9 & 10 & 11 \\
\hline Cynanchum acutum & 30.2 & - & - & - & - & - & - & - & - & - \\
\hline Poabulbosa & - & 56.7 & 16.0 & - & - & - & - & - & 9.9 & - \\
\hline Polygonum species & - & 55.7 & - & - & - & - & - & - & - & - \\
\hline Halimione verrucifera & - & 55.7 & - & 3.7 & 14.8 & - & - & - & 7.0 & - \\
\hline Bassia sedoides & - & 52.3 & - & - & - & - & - & 19.6 & - & - \\
\hline Elymus elongatus & - & 50.1 & - & - & - & - & - & - & - & - \\
\hline Petrosimonia oppositifolia & - & 48.2 & - & - & - & - & - & - & 22.8 & - \\
\hline Elymus repens & - & 46.3 & 7.4 & - & - & - & - & - & - & - \\
\hline Puccinellia festuciformis s. convoluta & - & 35.6 & - & 18.9 & 12.1 & - & 3.5 & - & 11.8 & 13.4 \\
\hline Bromus squarrosus & - & 33.3 & 13.9 & - & - & - & 4.9 & - & - & - \\
\hline Juncus gerardi & - & 30.8 & - & - & 19.8 & - & 10.5 & - & - & - \\
\hline Bassia hirsuta & - & - & 77.4 & 2.8 & - & 2.3 & - & - & - & - \\
\hline Atriplex prostrata & - & - & 39.6 & - & - & - & 5.9 & 13.3 & - & - \\
\hline Lepidium ruderale & - & - & 38.5 & - & - & - & - & - & - & - \\
\hline Ofaiston monandrum & - & - & - & 99.3 & - & - & - & - & - & - \\
\hline Pholiurus pannonicus & - & 7.3 & - & 78.9 & - & - & - & - & - & - \\
\hline Frankenia hirsuta & - & 2.8 & - & 53.2 & 18.0 & - & - & - & - & - \\
\hline Tetradiclis tenella & - & - & - & 48.0 & - & - & - & - & - & - \\
\hline Limonium suffruticosum & - & - & - & 42.1 & 9.6 & - & 8.8 & - & - & - \\
\hline Bassia prostrata & - & - & - & 40.6 & - & - & - & - & - & - \\
\hline Salsola laricina & - & - & - & 39.6 & 8.7 & - & 0.7 & - & - & - \\
\hline Halimione pedunculata & 3.6 & 16.0 & 5.9 & - & 52.9 & - & 6.4 & - & - & - \\
\hline Puccinellia distans & - & - & - & - & 43.5 & 3.9 & 9.2 & - & - & - \\
\hline Artemisia taurica & 2.2 & - & - & - & 38.1 & - & 3.8 & - & - & - \\
\hline Agrostis gigantea s. maeotica & - & - & - & - & 35.1 & - & - & - & - & - \\
\hline Suaeda baccifera & - & - & - & - & 31.4 & - & - & - & - & - \\
\hline Lepidium syvaschicum & - & - & - & - & 28.5 & - & 1.7 & - & - & - \\
\hline Cardaria draba & - & - & - & - & 27.1 & - & - & - & - & - \\
\hline Saussurea salsa & - & - & - & - & - & - & 39.1 & - & - & - \\
\hline Spergularia marina & - & - & 5.3 & - & 15.7 & - & 27.3 & - & - & - \\
\hline Cakile maritima s. euxina & - & - & - & - & - & - & - & 39.1 & - & - \\
\hline Puccinellia gigantea & 7.4 & - & 18.7 & - & - & 10.4 & - & 31.2 & - & - \\
\hline Aeluropus littoralis & - & - & 22.7 & - & 2.4 & - & 18.7 & 26.5 & - & - \\
\hline Apera spica-venti s. maritima & - & - & - & - & 14.5 & - & 1.0 & 25.6 & - & - \\
\hline Lepidium cartilagineum s. cartilagineum & - & - & - & - & - & - & - & - & 91.1 & - \\
\hline Cerastium pumilum s. pumilum & - & - & - & - & - & - & - & - & 44.4 & - \\
\hline Lepidium campestre & - & - & - & - & - & - & - & - & 36.1 & - \\
\hline Palimbia rediviva & - & - & - & - & - & - & - & - & 36.1 & - \\
\hline Scorzonera laciniata & - & - & - & - & - & - & - & - & 36.1 & - \\
\hline Vicia lathyroides & - & - & - & - & - & - & - & - & 36.1 & - \\
\hline Anthemis cotula & - & - & - & - & - & - & - & - & 36.1 & - \\
\hline Cerastium pumilum s. glutinosum & - & - & - & - & - & - & 8.5 & - & 29.4 & - \\
\hline Erophila verna & - & - & - & - & - & - & - & - & 25.4 & - \\
\hline Ranunculus marginatus v. trachycarpus & - & - & - & - & - & - & - & - & 25.4 & - \\
\hline Petrosimonia brachiata & - & - & - & - & - & - & - & - & - & 99.3 \\
\hline Atriplex calotheca & - & - & - & - & - & 3.5 & - & - & - & 66.1 \\
\hline Salsola kali s. tragus & - & - & - & - & - & - & - & - & - & 55.7 \\
\hline Petrosimonia triandra & - & - & - & - & - & - & - & - & - & 55.7 \\
\hline Artemisia santonicum & - & 14.0 & - & 0.4 & 9.1 & - & - & - & 3.7 & 50.1 \\
\hline Salsola soda & - & - & - & 4.0 & - & - & 10.8 & - & - & 38.8 \\
\hline Gypsophila perfoliata & - & 30.9 & 34.7 & - & - & - & - & - & - & - \\
\hline Limonium czurjukiense & - & 29.2 & - & 26.7 & 15.3 & - & - & - & - & - \\
\hline Limonium meyeri & - & 25.8 & 10.5 & 0.3 & 3.1 & - & 27.2 & - & - & - \\
\hline Limonium bellidifolium & 10.8 & - & - & 32.4 & 26.3 & - & 4.7 & - & - & - \\
\hline Suaeda maritima s. salsa & - & - & 2.8 & 21.4 & - & 48.1 & 48.1 & - & - & - \\
\hline Suaeda confusa & - & - & - & - & - & - & - & 54.4 & 17.0 & 54.4 \\
\hline Limonium gmelinii & - & - & - & - & - & - & - & - & 38.0 & 39.8 \\
\hline Camphorosma monspeliaca s. monspeliaca & - & 35.2 & - & - & - & - & - & - & 33.3 & 53.0 \\
\hline Atriplex tatarica & - & 13.7 & - & - & 5.8 & 0.5 & 18.6 & - & - & 23.4 \\
\hline Suaeda prostrata & - & - & - & 16.9 & 12.2 & - & - & - & 16.2 & 8.6 \\
\hline Bromus sterilis & 7.7 & - & - & - & - & - & - & - & - & - \\
\hline Lythrum volgense & 7.7 & - & - & - & - & - & - & - & - & - \\
\hline Suaeda altissima & 11.0 & - & - & - & - & - & - & - & - & - \\
\hline Taraxacum bessarabicum & 11.0 & - & - & - & - & - & - & - & - & - \\
\hline Calamagrostis epigejos & 11.0 & - & - & - & - & - & - & - & - & - \\
\hline Xanthium strumarium & - & - & - & - & - & - & 12.9 & - & - & - \\
\hline Polygonum maritimum & - & - & - & - & - & - & 12.9 & - & - & - \\
\hline Conyza canadensis & - & - & - & - & - & - & 12.9 & - & - & - \\
\hline Crepis sancta & - & - & - & - & - & - & 12.9 & - & - & - \\
\hline Salsola acutifolia & 13.4 & - & - & - & - & - & - & - & - & - \\
\hline Althaea officinalis & 2.8 & - & - & - & - & - & 10.6 & - & - & - \\
\hline
\end{tabular}




\begin{tabular}{|c|c|c|c|c|c|c|c|c|c|c|}
\hline 1 & 2 & 3 & 4 & 5 & 6 & 7 & 8 & 9 & 10 & 11 \\
\hline Carex extensa & 15.5 & - & - & - & - & - & - & - & - & - \\
\hline Bromus hordeaceus s. hordeaceus & - & - & - & - & 15.6 & - & - & - & - & - \\
\hline Artemisia austriaca & - & - & - & - & 15.6 & - & - & - & - & - \\
\hline Odontites verna s. serotina & 17.3 & - & - & - & - & - & - & - & - & - \\
\hline Cerastium semidecandrum & - & - & - & - & 11.1 & - & 6.9 & - & - & - \\
\hline Alyssum desertorum & - & - & - & - & 22.1 & - & - & - & - & - \\
\hline Puccinellia festuciformis & - & - & - & - & 22.1 & - & - & - & - & - \\
\hline Plantago tenuiflora & - & - & - & - & 22.1 & - & - & - & - & - \\
\hline Agropyron desertorum & - & - & - & - & - & - & 22.4 & - & - & - \\
\hline Triglochin maritima & 23.3 & - & - & - & - & - & - & - & - & - \\
\hline Cynodon dactylon & 0.2 & - & - & - & - & - & 20.9 & - & - & - \\
\hline Limonium tomentellum & 0.2 & - & - & - & - & - & 20.9 & - & - & - \\
\hline Polygonum arenarium s. arenarium & - & - & - & 23.8 & - & - & - & - & - & - \\
\hline Melilotus alba & 2.6 & - & - & - & - & - & 19.6 & - & - & - \\
\hline Spergularia media & 23.7 & - & - & - & - & - & 2.9 & - & - & - \\
\hline Crypsis aculeata & - & - & - & - & - & 24.7 & 2.1 & - & - & - \\
\hline Frankenia pulverulenta & - & - & - & - & 20.6 & - & 7.4 & - & - & - \\
\hline Scirpus maritimus s. maritimus & 18.1 & - & - & 11.9 & - & - & 0.5 & - & - & - \\
\hline Aster tripolium s. pannonicus & 6.3 & - & - & - & 23.9 & - & 6.7 & - & - & - \\
\hline Plantago maritima & 6.8 & - & - & 7.3 & - & - & 24.9 & - & - & - \\
\hline Juncus maritimus & - & - & - & - & 7.9 & 9.7 & 23.1 & - & - & - \\
\hline Phragmites australis & 7.4 & - & 15.7 & - & - & - & 8.7 & 13.7 & - & - \\
\hline Halocnemum strobilaceum & - & 12.1 & - & 14.8 & 4.6 & 2.4 & 2.6 & - & - & - \\
\hline Aster tripolium & 23.3 & - & 9.5 & - & - & - & 6.6 & 14.9 & - & - \\
\hline Salicornia prostrata & 22.5 & - & 15.9 & 18.0 & 12.7 & 22.5 & - & 10.5 & - & - \\
\hline
\end{tabular}

Note: numbers mark syntaxa: 1 - Salicornietum prostratae; 2 - Petrosimonio oppositifoliae-Salicornietum; 3 - Bassietum hirsutae; 4 - Ofaisto monandri-Salicornietum; 5 - Halimionetum pedunculatae; 6 - Salicornio perennantis-Suaedetum salsae; 7 - Suaedetum salsae; 8 - Suaedetum confusae; 9 - Lepidietum crassifoliae; 10 - Petrosimonio brachiatae-Artemisietum santonicae.

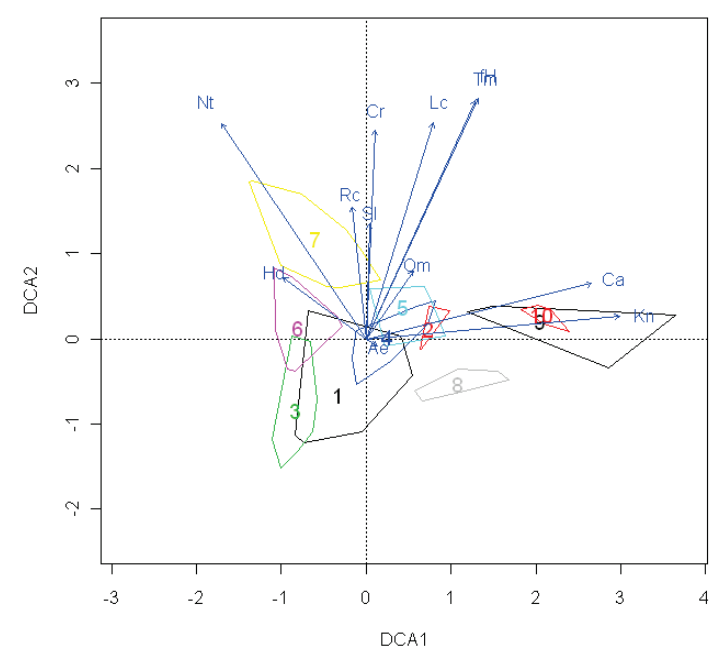

Fig. 10. Results of ordination analysis of the Therosalicornietea associations: the numbers mark syntaxa: 1 -Salicornietum prostratae; 2-Petrosimonio oppositifoliae-Salicornietum; 3 -Bassietum hirsutae; 4-Ofaisto monandri-Salicornietum; 5-Halimionetum pedunculatae; 6-Salicornio perennantis-Suaedetum salsae; 7 -Suaedetum salsae; 8 -Suaedetum confusae; 9 - Lepidietum crassifoliae; 10 - Petrosimonio brachiatae-Artemisietum santonicae

The class Crypsietea aculeatae includes ephemeral comparatively species-poor obligate halophytic and weakly nitrophilic pioneer plant communities that grow on open slightly alkaline soils with rapid changes in humidity and total salt regime. The class numbers 4 associations (Fig. 11, Table 5). They are widespread on the banks of slightly saline and saline water bodies (estuaries, lakes, local natural or anthropogenic depressions) in the steppe zone. Their territorial differentiation is determined by the moisture-drainage regime, total salt regime, and type of the soil. The alliance Cypero-Spergularion salinae includes phytocoenoses with the predominance of Crypsis schoenoides on wet saline habitats, mainly in natural or anthropogenic depressions. The alliance Heleochloion schoenoidis includes plant communities with the predominance of Crypsis schoenoides of negative relief forms of natural (pods) or non-natural origin on saline ecotopes. The alliance Lepidion latifolii is the vegetation with the predominance of Lepidium latifolium on saline territories that are periodically flooded and are located in the continental regions of Eastern Europe.

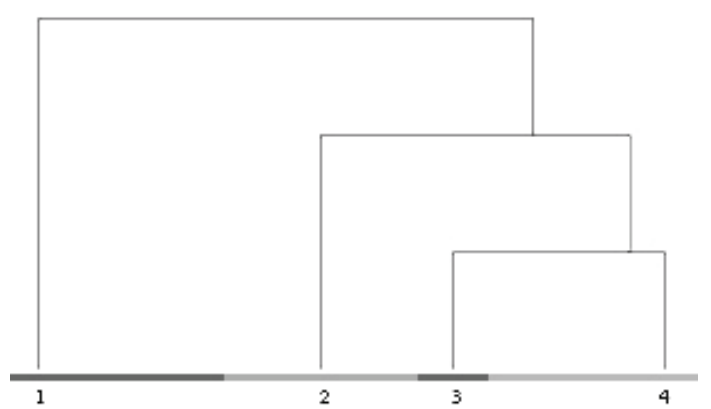

Fig. 11. Hierarchical cluster analysis of Crypsietea aculeatae phytosociological relevés: 1 -association Cynancho acutae-Lepidietum latifolii, 2 - association Crypsietum aculeatae, 3 - association Heleochloëtum schoenoidis, 4-association Polygono salsuginei-Crypsietum aculeatae

Table 5

Synoptic table of the class Crypsietea aculeatae

\begin{tabular}{lcccc}
\hline \multicolumn{1}{c}{ No. of syntaxa } & 1 & 2 & 3 & 4 \\
\hline \multicolumn{1}{c}{ Number of relevés } & 11 & 4 & 20 & 21 \\
\hline Taraxacum bessarabicum & 2 & 3 & 4 & 5 \\
Salicornia prostrata & 68.8 & - & - & - \\
Scirpus maritimus s. maritimus & 67.7 & - & - & - \\
Puccinellia festuciformis s. festuciformis & 64.5 & - & - & - \\
Suaeda prostrata & 62.0 & - & - & - \\
Triglochin maritima & 55.6 & - & - & 7.6 \\
Spergularia marina & 54.8 & - & - & - \\
Halimione pedunculata & 54.8 & - & - & - \\
Agrostis stolonifera & 53.2 & - & - & - \\
Juncus gerardi & 46.9 & - & - & - \\
Limonium bellidifolium & 33.7 & 15.5 & - & - \\
Sonchus arvensis & 26.4 & - & - & - \\
Scorzonera parviflora & 26.4 & - & - & - \\
Limonium meyeri & 26.4 & - & - & - \\
Camphorosma annua & 26.4 & - & - & - \\
Polygonum aviculare & 26.4 & - & - & - \\
Crypsis schoenoides & - & 83.2 & - & - \\
Echinochloa crus-galli & - & 70.7 & 23.6 & - \\
Puccinellia distans & - & 65.5 & - & - \\
Atriplex prostrata & - & 64.7 & 6.6 & - \\
Xanthium strumarium s. italicum & - & 60.3 & - & - \\
Taraxacum officinale & - & 44.7 & - & - \\
Plantago major s. intermedia & - & 44.7 & - & - \\
& - & 44.7 & - & - \\
Dono & & & & \\
\hline & & & & - \\
\hline & & &
\end{tabular}




\begin{tabular}{|c|c|c|c|c|}
\hline 1 & 2 & 3 & 4 & 5 \\
\hline Daucus carota & - & 44.7 & - & - \\
\hline Limonium tomentellum & - & 44.7 & - & - \\
\hline Pholiurus pannonicus & - & 44.7 & - & - \\
\hline Ambrosia artemisiifolia & - & 44.7 & - & - \\
\hline Lepidium ruderale & - & 44.7 & - & - \\
\hline Artemisia santonicum & - & 34.2 & - & 18.2 \\
\hline Beckmannia eruciformis & - & 33.2 & 2.6 & - \\
\hline Atriplex tatarica & - & 29.4 & - & 8.7 \\
\hline Limonium gmelinii & - & - & 100.0 & - \\
\hline Polygonum salsugineum & - & - & 100.0 & - \\
\hline Rumex stenophyllus & - & - & 86.6 & - \\
\hline Eleocharis palustris & - & - & 65.5 & - \\
\hline Alisma lanceolatum & - & - & 65.5 & - \\
\hline Salsola kali s. tragus & - & - & 57.0 & - \\
\hline Juncus maritimus & - & - & 48.8 & - \\
\hline Typha laxmannii & - & - & 44.7 & - \\
\hline Ventenata dubia & - & - & 27.7 & - \\
\hline Lepidium latifolium & - & - & - & 100.0 \\
\hline Cynanchum acutum & - & - & - & 73.2 \\
\hline Lactuca tatarica & - & - & - & 66.7 \\
\hline Sinapis alba & - & - & - & 63.7 \\
\hline Atriplex littoralis & - & - & - & 63.7 \\
\hline Bromus squarrosus & - & 4.6 & - & 56.3 \\
\hline Polygonum arenarium s. arenarium & - & - & - & 56.2 \\
\hline Bassia hirsuta & 2.7 & - & - & 48.5 \\
\hline Plantago arenaria & - & - & - & 48.0 \\
\hline Bassia sedoides & - & - & - & 48.0 \\
\hline Melilotus alba & - & - & - & 48.0 \\
\hline Elymus repens & - & 4.7 & - & 43.1 \\
\hline Polygonum scythicum & - & - & - & 38.7 \\
\hline Argusia sibirica & - & - & - & 38.7 \\
\hline Erysimum repandum & - & - & - & 33.3 \\
\hline Elymus elongatus & - & - & 19.9 & 32.4 \\
\hline Plantago maritima & - & - & - & 27.1 \\
\hline Bromus japonicus & - & - & - & 27.1 \\
\hline Chondrilla juncea & - & - & - & 27.1 \\
\hline Puccinellia gigantea & - & - & - & 27.1 \\
\hline Crypsis aculeata & 57.7 & - & 57.7 & - \\
\hline Aster tripolium & 29.4 & 50.6 & - & - \\
\hline Centaurea apiculata s. adpressa & - & - & - & 19.0 \\
\hline Cuscuta monogyna & - & - & - & 19.0 \\
\hline Agrostis gigantea & - & - & - & 19.0 \\
\hline Crambe maritima & - & - & - & 19.0 \\
\hline Eleocharis uniglumis & - & - & - & 19.0 \\
\hline Leymus racemosus s. sabulosus & - & - & - & 19.0 \\
\hline Seseli tortuosum & - & - & - & 19.0 \\
\hline Phragmites australis & 17.5 & - & - & 9.7 \\
\hline Pulicaria dysenterica & - & - & 19.5 & - \\
\hline Pulicaria paludosa & - & - & 19.5 & - \\
\hline
\end{tabular}

Notes: the numbers mark syntaxa: 1 -Crypsietum aculeatae; 2 - Heleochloëtum schoenoidis; 3 - Polygono salsuginei-Crypsietum aculeatae; 4 - Cynancho acutae-Lepidietum latifolii.

DCA-ordination results of the Crypsietea aculetae plant communities showed that they differentiate mostly along the gradients of nitrogen content in the soil and variability of damping, vectors of which are the closest to the first ordination axis (Fig. 12). Additionally, the leading factors for Crypsietum aculeatae plant communities are the total salt regime and soil acidity, for Heleochloëtum schoenoidis coenoses - cryoclimate.

\section{Discussion}

Therefore, the syntaxonomic structure of Cakiletea maritimae class in Ukraine includes 4 associations, which belong to 1 order and 1 alliance; Ammophiletea have 8 associations, 1 order and 1 alliance; Crithmo-Staticetea -1 order, 2 alliances and 3 associations. Like both western and eastern European coastal vegetation of these classes, it is marked by a low level of phytocoenotic diversity (Sanda et al., 2008; Tzonev et al., 2009; Biondi et al., 2014) which is caused by extreme conditions of these habitats. The specificity of coastal plant communities in Ukraine is identified on the associations-rank level and conditioned by the action and intensity of coastal processes of the Ukrainian part of the Black and Azov Sea as well as the climatic conditions of the region. The communities of Kochio prostratae-Limonion meyeri Korzhenevsky et Klyukin 1987 and Elytrigio
bessarabicae-Lactucion tataricae Korzhenevsky ex Didukh et Mucina in Mucina et al. 2016 alliances are typical only for the Crimean Peninsula.

The classes of Therosalicornietea and Crypsietea aculeatae are also characterized by middle European level of phytocoenotic diversity (Borhidi, 2003; Chytrý, 2007; Sanda et al., 2008). Crypsietea aculeatae numbers 1 order, 3 alliances and 4 associations, Therosalicornietea - 1 order, 2 alliances and 10 associations.

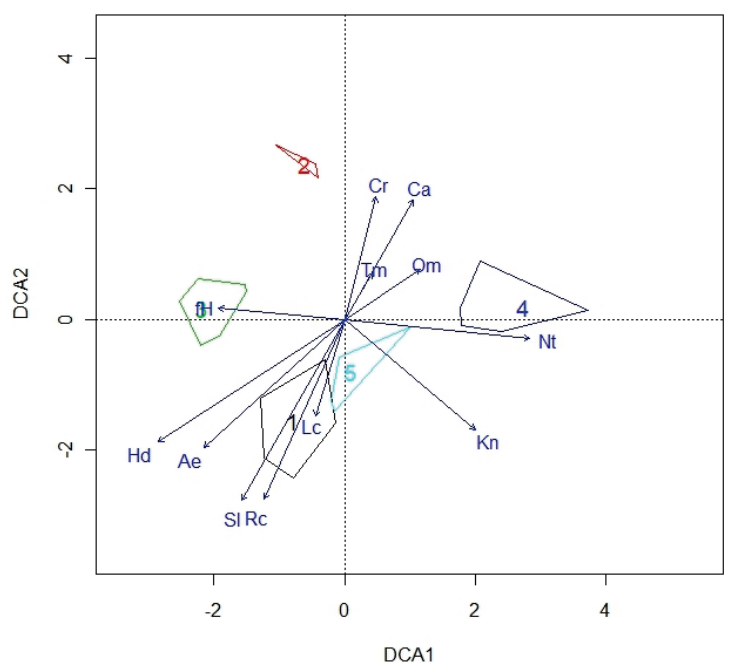

Fig. 12. Results of ordination analysis of the Crypsietea aculeatae associations: 1-Crypsietum aculeatae; 2 - Heleochloëtum schoenoidis; 3-Polygono salsuginei-Crypsietum aculeatae;
4-Cynancho acutae-Lepidietum latifolii

Today the question of Isoëto-Nanojuncetea and Crypsietea aculeatae plant communities' correlation is being discussed in Europe. Italian, Spanish and French phytosociologists consider the class Crypsietea aculeatae as a syntaxomic synonym of Isoëto-Nanojuncetea (Rivas-Martínez et al., 2001; Bardat et al., 2004; Biondi et al., 2014). Bulgarian scientists include syntaxa of the order Crypsietalia aculeatae to belong to the IsoëtoNanojuncetea class (Tzonev et al., 2009). Rodwell et al. (2002) share the same point of view, identifying three alliances within this class: CyperoSpergularion salinae, Polygono salsuginei-Crypsion aculeatae and Puccinellion peisonis. Romanian phytosociologists think that communities of the Cypero-Spergularion alliance belong to the Puccinellio-Salicornietea class, which has a wider conception for them (Sanda et al., 2008), and Hungarian scientists relate it to Thero-Suaedetea Vicherek 1973 class (Borhidi, 2003). Our studies showed that phytocoenoses of these two classes can be quite clearly distinguished by the total salt regime and diagnostic species.

The most discussed questions about the conception and structure of the Therosalicornietea class relate to the taxonomy of the genus Salicornia. Depending on the distribution of its species (Salicornia pusilla J. Woods, S. ramosissima J. Woods, S. europaea L., S. obscura P. W. Ball \& Tutin, S. nitens P. W. Ball \& Tutin, S. fragilis P. W. Ball \& Tutin, S. dolichostachya Moss, S. procumbens Smith) (Davy et al., 2001), several alliances are identified on the territory of Europe (Mucina et al., 2016). However, class Thero-Suaedetea is distinguished in Hungary, Italy, Spain, and France. In Romania pioneer communities of the order Salicornietalia Br.Bl. (1928) 1933 on solonchaks belongs to the Puccinellio-Salicornietea Țopa 1939 class (Sanda et al., 2008).

Numerous studies confirm the importance of the soil humidity, edaphic and climatic characteristics of the habitats (Jarvis et al., 2016; Kuzemko et al., 2016; Pinto et al., 2016; Ermakov et al., 2017; Bagrikova, 2018), that affect phytodiversity and structure of pioneer vegetation. We agree with the assertion that the ecological specificity of a pioneer plant communities is driven by a combination of several factors (Chytrý et al., 2007, 2013; Didukh, 2012; Didukh et al., 2015). However, for the pioneer plant communities, considerable influence is exerted by the extremality of the environmental conditions. This involves first of all their irregularity such as the mechanical action of the sea surf and the impact of waves, the dynamic processes of denudation and accumulation, total salt regime, as 
well as the extremely variable regime of soil humidity. In this aspect, it is possible to make parallels with the variability of ecological gradients for subalpine vegetation, which also develops in extreme ecotopes (Čarni et al., 2011; Dyakov, 2014). These features cause the extreme vulnerability of pioneer vegetation to biotic and abiotic changes (Dubyna et al., 2019), determine the weakness of its coenotic connections and low anthropotolerance (Dubyna et al., 2015, 2016a, b), which requires immediate action for conservation of this specific and unique type of phytocenosis.

\section{Conclusion}

Littoral and halophytic pioneer vegetation in Ukraine of Cakiletea maritimae, Ammophiletea, Crithmo-Staticetea, Therosalicornietea and Crypsietea aculeatae classes are represented by 29 associations, 9 alliances and 5 orders. The leading factors of territorial and ecological differentiation of littoral and halophytic pioneer vegetation in Ukraine have been identified. It has been determined that the intensity of abrasive-accumulative action of the sea, soil type, and its density explains the territorial distribution of Ammophiletea, Cakiletea maritimae and Crithmo-Staticetea. At the same time, relief of ecotopes, soil structure and period of flooding have a great impact on Therosalicornietea and Crypsietea aculeatae plant communities.

The conducted ordination analysis of coastal and halophytic pioneer vegetation in Ukraine at the classes and alliances-rank levels has shown that the leading factors of its ecological differentiation are soil humidity and soil aeration as well as nitrogen content. On the basis of the results of ordination of syntaxa within the classes, we have defined the main factors that correlate with the specifics of the habitats where the pioneer vegetation evolves. It has been found that the ecological differentiation within the Ammophiletea class is caused by the variability of damping, Therosalicornietea - by the total salt regime, soil acidity as well as continental climate and cryoclimate, Cakiletea maritimae - by the variability of damping, cryoclimate, soil acidity, nitrogen and carbonate content in the soil, Crithmo-Staticetea - by the light regime and climate humidity, and Crypsietea aculeatae - by the nitrogen content in the soil and its aeration.

These studies give the opportunity to determine the place of the syntaxa of pioneer vegetation in Ukraine within the European Vegetation Classification. The results of the conducted research can be useful for the formation of the preservation and ecological network in Ukraine, compilation of The Prodrome of the Vegetation of Europe, the next volumes of the editions "Vegetation of Ukraine" and "Green Data Book of Ukraine", for further research of succession and adaptation processes in rapidly changing environmental conditions, establishment of development patterns in regeneration geological systems as well as identifying the ways to manage them.

\section{References}

Bagrikova, N. A. (2018). Differentsiatsiya soobshchestv segetalnoy rastitelnosti Kryma na gradientakh faktorov sredy [Differentiation of segetal communities of Crimea on environmental factors gradients]. Science in the South of Russia, 14(2), 73-87 (in Russian).

Bairak, O. M. (1997). Syntaksonomiya halofilnoi roslynnosti Livoberezhnoho Prydniprov'ya [Syntaxonomy of halophilic vegetation of the Left Bank Dnieper]. Ukrainian Phytosociological Collection, Series A, 2(7), 68-74 (in Ukrainian).

Bardat, J., Bioret, F., Botineau, M., Boullet, V., Delpech, R., Genu, J.-M., Haury, J., Lacoste, A., Rameau, J.-C., Royer, J.-M., Roux, G., \& Touffet, J. M. (2004). Prodrome des Végétations de France. MNHN. Patrimoines Naturel. Paris.

Berg, C., Dengler, J., Abdank, A., \& Iermann, M. (Eds.). (2004). Die Pflanzengesellschaften Mecklenburg-Vorpommerns und ihre Gefährdung. Wiessdorn Verlag, Jena.

Biondi, E., Blasi, C., Allegrezza, M., Anzellotti, I., Azzella, M. M., Carli, E., Casavecchia, S., Copiz, R., Del Vico, E., Facioni, L., Galdenzi, D., Gasparri, R., Lasen, C., Pesaresi, S., Poldini, L., Sburlino, G., Taffetani, F., Vagge, I., \& Zivkovic, L. (2014). Plant communities of Italy: The Vegetation Prodrome. Plant Biosystems, 148(4), 728-814.

Borhidi, A. (2003). Magyarorszag növćnytársulásai. Akadémiai Kiadó, Budapest.

Buynevich, I., Yanko-Hombach, V., Gilbert, A. S., \& Martin, R. E. (Eds.). (2011). Geology and geoarchaeology of the Black Sea region: Beyond the flood hypothesis. The Geological Society of America, Colorado.
Cao, G., Tsuchiya, K., Zhu, W., \& Okuro, T. (2019). Vegetation dynamics of abandoned paddy fields and surrounding wetlands in the lower Tumen River Basin, Northeast China. PeerJ, 7, e6704.

Čarni, A., Juvan, N., Košir, P., Marinšek, A., Paušič, A., \& Šilc, U. (2011). Plant communities in gradients. Plant Biosystems, 145, 54-64.

Chytrý, M. (Ed.). (2007). Vegetace České republiky. 1. Travinná a keřičková vegetace [Vegetation of the Czech Republic. 1. Grassland and Heathland Vegetation]. Academia, Praha (in Czech).

Chytrý, M. (Ed.). (2013). Vegetace České republiky. 4. Lesní a křovinná vegetace [Vegetation of the Czech Republic. 4. Forest and Scrub Vegetation]. Academia, Praha (in Czech).

Çoban, S., \& Willner, W. (2019). Numerical classification of the forest vegetation in the Western Euxine Region of Turkey. Phytocoenologia, 49(1), 71-106.

Correa-Metrio, A., Dechnik, Y., Lozano-García, S., \& Caballero, M. (2014). Detrended correspondence analysis: A useful tool to quantify ecological changes from fossil data sets. Boletin de la Sociedad Geologica Mexicana, 66(1), 135-143.

Davy, A. J., Bishop, G. F., \& Costa, C. S. B. (2001). Salicornia L. (Salicornia pusilla J. Woods, S. ramosissima J. Woods, S. europaea L., S. obscura P. W. Ball \& Tutin, S. nitens P. W. Ball \& Tutin, S. fragilis P. W. Ball \& Tutin and S. dolichostachya Moss). Journal of Ecology, 89, 681-707.

Del Vecchio, S., Fantinato, E., Janssen, J., Bioret, F., Acosta, A., Prisco, I., Tzonev, R., Marcenò, C., Rodwell, J., \& Buffa, G. (2018). Biogeographic variability of coastal perennial grasslands at the European scale. Applied Vegetation Science, 21(2), 312-321.

Didukh, Y. P. (2011). The ecological scales for the species of Ukrainian flora and their use in synphytoindication. Phytosociocentre, Kyiv.

Didukh, Y. P. (2012). Osnovy bioindykatsii [Fundamentals of bioindication]. Naukova Dumka, Kyiv (in Ukrainian).

Didukh, Y. P., Chusova, O. O., Olshevska, I. A., \& Polishchuk, Y. V. (2015). River valleys as the object of ecological and geobotanical research. Ukrainian Botanical Journal, 72(5), 415-430.

Dubyna, D. V., \& Dziuba, T. P. (2005). Phytocenotic diversity of Dzharylgach Island (Kherson region). Ukrainian Botanical Journal, 62(2), 255-269 (in Ukrainian).

Dubyna, D. V., \& Neuhäuslová, Z. (2003). The vegetation of the Azov-Sivaš National Nature Park. Class Thero-Salicornietea (S. Pignatti 1953) R. Tx. in R. Tx. et Oberdorfer 1958. Thaiszia Journal of Botany, 13(1), 1-30.

Dubyna, D. V., Dvoretskyi, T. V., Dziuba, T. P., \& Tymoshenko, P. A. (2016a). Biomorfolohichna struktura tsenoflor pionernoi roslynnosti Ukrainy [Biomorphological structure of coenofloras of pioneer vegetation of Ukraine]. Chornomorski Botanical Journal, 12(2), 107-123 (in Ukrainian).

Dubyna, D. V., Dziuba, T. P., Davydov, D. A., \& Iemelianova, S. M. (2015). Suchasnyi stan syntaksonomii ta aktualni zavdannya vyvchennya pionernoi roslynnosti Ukrainy [Contemporary status of syntaxonomy and current tasks of the research on pioneer vegetation in Ukraine]. Ukrainian Botanical Journal, 72(6), 527-541 (in Ukrainian).

Dubyna, D. V., Dziuba, T. P., Iemelianova, S. M., \& Davydov, D. A. (2016b). Suchasnyi stan ta aktualni zavdannya okhorony pionernoi roslynnosti Ukrainy [Contemporary state and actual tasks of protection of pioneer vegetation in Ukraine]. Ukrainian Botanical Journal, 73(1), 11-20 (in Ukrainian).

Dubyna, D. V., Dziuba, T. P., Iemelianova, S. M., Bagrikova, N. O., Borysova, O. V., Borsukevych, L. M., Vynokurov, D. S., Gapon, S. V., Gapon, Y. V., Davydov, D. A., Dvoretsky, T. V., Didukh, Y. P., Zhmud, O. I., Kozyr, M. S., Konyschuk, V. V., Kuzemko, A. A., Paskevych, N. A., Ryff, L. E., Solomakha, V. A., FelbabaKlushyna, L. M., Fitsailo, T. V., Chorna, G. A., Chomey, I. I., Shelyag-Sosonko, Y. R., \& Iakushenko, D. M. (2019). Prodromus roslynnosti Ukrainy [Prodrome of the vegetation of Ukraine]. Naukova Dumka, Kyiv (in Ukrainian).

Dubyna, D. V., Dziuba, T. P., Neuhäuslová, Z., Solomakha, V. A., Tyshchenko, O. V., \& Shelyag-Sosonko, Y. R. (2007b). Halofitna roslynnist. Klasy Bolboschoenetea maritimi, Festuco-Puccinellietea, Molinio-Juncetea, Crypsietea aculeatae, Thero-Salicornietea strictae, Salicornietea fruticosae, Juncetea maritimi [Halophytic vegetation. Bolboschoenetea maritimi, Festuco-Puccinellietea, Molinio-Juncetea, Crypsietea aculeatae, Thero-Salicornietea strictae, Salicornietea fruticosae, Juncetea maritimi Classes]. In: The vegetation of Ukraine. Phytosociocentre, Kyiv (in Ukrainian).

Dubyna, D. V., Iemelianova, S. M., Dvoretzkiy, T. V., Dziuba, T. P., Tymoshenko, P. A. (2019). Adventyzatsiya tsenoflor klasiv pionernoi roslynnosti Ukrainy [Adventization of coenofloras of the classes of pioneer vegetation in Ukraine]. Ukrainian Botanical Journal, 76(5), 499-510 (in Ukrainian).

Dubyna, D. V., Neuhäuslová, Z., \& Shelyag-Sosonko, Y. R. (1995). Vegetation of the "Birjucij Island" spit in Azov Sea. Sand steppe vegetation. Folia Geobotanica et Phytotaxonomica, 30, 1-31.

Dubyna, D. V., Neuhäuslová, Z., Dziuba, T. P., \& Shelyag-Sosonko, Y. R. (2004). Klasyfikatsiya ta prodromus syntaksonomichnoi riznomanitnosti vodoym, perezvolozhenykh terytoriy ta aren Pivnichnoho Prychomomor'ya [Classification and prodromus of vegetation of reservoirs, overmoisturized territories and arena of the Northern part of the Black Sea region]. Phytosociocentre, Kyiv (in Ukrainian). 
Dubyna, D. V., Shelyag-Sosonko, Y. R., Zhmud, O. I., Dvoretskiy, T. V., Dziuba, T. P., \& Tymoshenko, P. A. (2003). Dunayskyi biosfernyi zapovidnyk. Roslynnyi svit [Dunaisky Biosphere Reserve. Plant Kingdom]. Phytosociocentre, Kyiv (in Ukrainian).

Dubyna, D. V., Tymoshenko, P. A., \& Golub, V. B. (2007a). Syntaksonomiya roslynnosti prymorsko-dyunnykh ekosystem Ukrainy. Klasy Cakiletea maritimae i Ammophiletea [Syntaxonomy of vegetation of seaside dunes ecosystems of Ukraine. Cakiletea maritimae and Ammophiletea classes]. Chornomorski Botanical Journal, 3(2), 19-36 (in Ukrainian).

Dyakov, N. R. (2014). Gradient analysis of vegetation on the south slope of Vitosha Mountain, Southwest Bulgaria. Applied Ecology and Environmental Research, 12(4), 1003-1025.

Dziuba, T. P., Solomakha, T. D., \& Tymoshenko, P. A. (2010). Roslynni uhrupovannya [Vegetation communities]. Pereyaslav-Khmelnytskiy. Nature: vegetation. Critical inventory annotated notes of flora and vegetation: vascular plants, bryophytes, lichens, and algae. FOP Majdanchenko I. S., Korsun-Shevchenkivsky. Pp. 86-95 (in Ukrainian).

Ermakov, N. B., Larionov, A. V., Polyakova, M. A., \& Plugatar, Y. V. (2017). Ecological interpretation of higher units of steppe vegetation in the mountains of southern Middle Siberia by quantitative ordination. Biology Bulletin Reviews, 7(3), 229-237.

Hennekens, S. M., \& Schaminée, J. H. J. (2001). TURBOVEG, a comprehensive date base management system for vegetation data. Journal of Vegetation Science, 12, 589-591.

Hill, M. O. (1979). TWINSPAN - a FORTRAN program for arranging multivariate data in an ordered two-way table by classification of the individuals and the attributes. Ithaca, New York.

Hill, M. O., \& Gauch, H. G. (1980). Detrended correspondence analysis: An improved ordination technique. Vegetatio, 42, 47-58.

Jansons, A., Robalte, L., Čakšs, R., \& Matisons, R. (2016). Long-term effect of whole tree biomass harvesting on ground cover vegetation in a dry Scots pine stand. Silva Fennica, 50(5), 1661.

Jarvis, S., Rowe, E., Henrys, P., Smart, S. M., Jones, L., \& Garbutt, A. (2016). Empirical realised niche models for British coastal plant species. Journal of Coastal Conservation, 20(2), 107-116.

Korolyuk, A. Y., Lebedeva, M. V., Yamalov, S. M., Golovanov, Y. M., Dulepova, N. A., Zolotareva, N. V., \& Teptina, A. Y. (2018). The petrophytic steppes of the Urals: Diversity and ecological drivers. The Fourth International Scientific Conference on Ecology and Geography of Plants and Plant Communities, KnE Life Sciences. Pp. 88-94.

Korzhenevskiy, V. V., \& Kvitnitskaya, A. A. (2014). Sintaksonomiya rastitelnosti eolovogo rel'efa Kryma [Syntaxonomy of vegetation of the Crimean eolian relief]. Works of the State Nikitsky Botanical Gardens, 136, 41-55 (in Russian).

Korzhenevskiy, V. V., Bagrikova, N. A., Ryff, L. E., \& Levon, A. R. (2003). Prodromus rastitelnosti Kryma (20 let na platforme floristicheskoy klassifikatsii) [Prodromus of the Crimean vegetation (20 years on the platform of floristic classification)]. Bulletin Main Botanical Garden RAN, Yalta, 186, 32-51 (in Russian).

Kuzemko, A. A., Steinbauer, M. J., Becker, T., Didukh, Y. P., Dolnik, C., Jeschke, M., Naqinezhad, A., Ugurlu, E., Vassilev, K., \& Dengler, J. (2016). Patterns and drivers of phytodiversity of steppe grasslands of Central Podolia (Ukraine). Biodiversity and Conservation, 25(12), 2233-2250.

Lashchinskiy, N. N., Tishchenko, M. P., \& Korolyuk, A. Y. (2019). Kolichestvennyi analiz lokalnykh tsenoflor stepnoy zony Severnogo Kazakhstana [Quantitative analysis of local coenofloras in the steppe zone of Northern Kazakhstan]. Vestnik Tomskogo Gosudarstvennogo Universiteta, Biologiya, 45, 69-90 (in Russian).

Mucina, L., Bültman, H., Dierssen, K., Theurillat, J.-P., Dengler, J., Čarni, A., Šumberová, K., Raus, T., Di Pietro, R., Gavilán Garcia, R., Chytrý, M., Iakushenko, D., Schaminée, J. H. J., Bergmeier, E., Santos Guerra, A., Daniëls, F. J. A., Ermakov, N., Valachovič, M., Pigantti, S., Rodwell, J. S., Pallas, J., Capelo, J., Weber, H. E., Lysenko, T., Solomeshch, A., Dimopoulos, P., Aguiar, C., Freitag, H., Hennekens, S. M., \& Tichý, L. (2016). Vegetation of Europe: hierarchical floristic classification system of vascular plant, bryophyte, lichen, and algal communities. Applied Vegetation Science, 19(1), 3-264.

Pinto, P., Dupouey, J.-L., Hervé, J.-C., Legay, M., Wurpillot, S., Montpied, P., \& Gégout, J.-C. (2016). Optimizing the bioindication of forest soil acidity, nitrogen and mineral nutrition using plant species. Ecological Indicators, 71, 359-367.

Rivas-Martínez, S., Fernández-González, F., Loidi, J., Lousã, M., \& Penas, A. (2001). Syntaxonomical checklist of vascular plant communities of Spain and Portugal to association level. Itinera Geobotanica, 14, 5-341.
Rodwell, J. S., Schaminee, J. H. J., Mucina, L., Pignatti, S., Dring, J., \& Moss, D. (2002). The diversity of European Vegetation. An overview of phytosociological alliances and their relationships to EUNIS habitats. National Centre for Agriculture, Nature Management and Fisheries, Wageningen.

Roleček, J., Tichý, L., Zelený, D., \& Chytrý, M. (2009). Modified TWINSPAN classification in which the hierarchy respects cluster heterogeneity. Journal of Vegetation Science, 20, 596-602.

Rudenko, L. G. (Ed.). (2007). Natsionalnyi atlas Ukrainy [National Atlas of Ukraine]. Cartography, Kyiv (in Ukrainian).

Sanda, V., Öllerer, K., \& Burescu, P. (2008). Fitocenozele din Romănia. Sintaxonomia, structură, dinamică si evolutie. Ars Docendi, București (in Romanian).

Sergienko, L. A., \& Sonina, A. V. (2011). Osnovnye puti osvoeniya okolovodnykh severnykh territoriy vysshimi rasteniyami $\mathrm{i}$ lishaynikami [The main ways of the absorption of the near-water northern territories by higher plants and lichens]. Modern Problems of Science and Education, 6, 4 (in Russian).

Slezák, M., Hrivnák, R., \& Machava, J. (2017). Environmental controls of plant species richness and species composition in black alder floodplain forests of central Slovakia. Tuexenia, 37, 79-94.

Sörensen, T. A. (1948). A method of establishing groups of equal amplitude inplant sociology based on similary of species content, and its application to analyses of the vegetation of Danish commons. Kongelige Danske Videnskabernes Selskabs Biologiske Skrifter, 5, 1-34.

Ter Braak, C. J. F., \& Smilauer, P. (2015). Topics in constrained and unconstrained ordination. Plant Ecology, 216(5), 683-696.

The state of the marine environment: Trends and processes (2006). Reports and Studies, UNEP/GPA, The Hague.

Tichy, L. (2002). JUICE, software for vegetation classification. Journal of Vegetation Science, 13, 451-453.

Tutin, T. G. (Ed.) (1964-1980). Flora Europaea. Cambridge. Volume 1-5.

Tymoshenko, P. A. (1999). Suchasnyi stan klasyfikatsii roslynnosti aren pivdnya Ukrainy [The modern state of classification of vegetation of the aren of Southern Ukraine]. Ukrainian Phytosociological Collection, Series A, 1-2(12-13), 201205 (in Ukrainian).

Tyshchenko, O. V. (2006). Roslynnist prymorskykh kis pivnichnoho uzberezhzhya Azovskoho morya [The vegetation of the northern coastal spits of the Sea of Azov]. Phytosociocentre, Kyiv (in Ukrainian).

Tzonev, R. T., Dimitrov, M. A., \& Roussakova, V. H. (2009). Syntaxa according to the Braun-Blanquet approach in Bulgaria. Phytologia Balcanica, 15(2), 209-233.

Valachovič, M., Ot’ahelová, H., Stanová, V., \& Maglocký, Š. (1995). Rastlinné spoločenstvá Slovenska 1. Pionierska vegetácia [Plant communities of Slovakia 1. Pioneer vegetation]. Veda, Bratislava (in Slovenian).

Venables, W. N., \& Smith, D. M. (2008). An introduction to R. 2nd edition. Network Theory Ltd.

Vicherek, J. (1971). Grundriss einer Systematik der Strandgesellschaften des Schwarzen Meeres. Folia Geobotanica et Phytotaxonomica, 6, 127-145.

Vojtyuk, B. Y. (2005). Roslynnist zasolenykh hruntiv Pivnichno-Zakhidnoho Prychornomor'ya (suchasnyi stan, klasyfikatsiya, napryamky transformatsii, okhorona) [The Vegetation of the saline soils of the Northwestern part of the Black Sea Region (the modern state, classification, directions of transformations, and protection)]. Phytosociocentre, Kyiv (in Ukrainian).

Westhoff, V., \& van der Maarel, E. (1973). The Braun-Blanquet approach. 2nd ed. In: Whittaker, R. (Ed.). Classification of Plant Communities. Pp. 287-399.

Whittaker, R. H. (1978). Approaches to classifying vegetation on classification of plant communities. 2nd ed. Junk, The Hague.

Willner, W., Roleček, J., Korolyuk, A., Dengler, J., Chytrý, M., Janišová, M., Lengyel, A., Aćić, S., Becker, T., Ćuk, M., Demina, O., Jandt, U., Kącki, Z., Kuzemko, A., Kropf, M., Lebedeva, M., Semenishchenkov, Y., Šilc, U., Stančić, Z., Staudinger, M., Vassilev, K., \& Yamalov, S. (2019). Formalized classification of semi-dry grasslands in central and Eastern Europe. Preslia, 91, 25-49.

Willner, W., Tichy, L., \& Chytry, M. (2009). Effects of different fidelity measures and contexts on the determination of diagnostic species. Journal of Vegetation Science, 20, 130-137.

Yousaf, A., Shabbir, R., Jabeen, A., Erum, S., \& Ahmad, S. S. (2016). Linkage between herbaceous vegetation and soil characteristics along rawal dam Islamabad. Journal of Soil Science and Plant Nutrition, 16(1), 7.

Zhou, L., Shen, H., Chen, L., Li, H., Zhang, P., Zhao, X., Liu, T., Liu, S., Xing, A., Hu, H., \& Fang, J. (2019). Species richness and composition of shrub-encroached grasslands in relation to environmental factors in Northern China. Journal of Plant Ecology, 12(1), 56-66. 